\title{
Die Karlsbad-Reise des Ferdinand Krackowizer im Jahre 1909. Anmerkungen zu einer Kulturgeschichte der bürgerlichen Badereise
}

\author{
Elisabeth Fendl
}

DOI: 10.21104/CL.2020.4.01

Ferdinand Krackowizer's Trip to Karlovy Vary in 1909. A contribution to the history of the bourgeois bathing trip

\begin{abstract}
In May 1909, Ferdinand Krackowizer (1844-1933), an archival councillor from Linz, took a trip to the spa in Karlovy Vary. He kept a travel diary as well as a memory album where he stored all sorts of documents: postcards, concert programmes, tickets, menus, a report from his attending physician, etc. By analysing these documents we are able to reconstruct in detail Krackowizer's stay at the spa and gain deeper knowledge of the everyday spa culture in Karlovy Vary at the turn of the century. In addition, the analysis takes into account the opinions and assessments provided by the traveller himself in his travel diary. The diary further facilitates reconstructing Krackowizer's networks and understanding different aspects of cultural practices connected to spa travel. Finally, these primary sources show the importance which Krackowizer attributed to his spa trip as a form of external representation.
\end{abstract}

\section{Key words}

Spa tourism, bourgeois bathing trip, anti-Semitism, ego-documents, diary

\section{Contact}

Elisabeth Fendl, Institut für Volkskunde der Deutschen des östlichen Europa (IVDE), Goethestraße 63, 79100 Freiburg, Germany; e-mail: elisabeth.fendl@ivde.bwl.de.

\section{Jak citovat / How to cite}

Fendl, Elisabeth. (2020). Die Karlsbad-Reise des Ferdinand Krackowizer im Jahre 1909. Anmerkungen zu einer Kulturgeschichte der bürgerlichen Badereise. Český lid 107: 399-438. doi:http://dx.doi.org/10.21104/ CL.2020.4.01 


\section{Einleitung}

Im Mai 1909 unternahm Dr. Ferdinand Krackowizer (Wels 1844 - Linz 1933), Archivrat aus Linz, eine Badereise nach Karlsbad. Er hatte den Termin des Kuraufenthaltes so gelegt, dass er in Karlsbad seinen 65. Geburtstag begehen konnte. Krackowizer, der in Wien und Innsbruck Jus studiert hatte und 1868 in den oberösterreichischen Landesdienst getreten war, wurde 1875 Landesarchivar. 1903 ging er in Ruhestand. Er war kaiserlicher Rat, 1921 wurde ihm ehrenhalber der Titel „oberösterreichischer Landesarchivdirektor" verliehen. Seine 1890 bei Reclam in Leipzig erschienene „Naturgeschichte des österreichischen Studenten“ machte ihn als „humoristische[n] Schriftsteller [...] über die Grenzen seiner Heimat bekannt" (ÖBL 1967). Daneben verfasste er eine große Zahl landes- und heimatkundlicher Schriften und trat als Herausgeber des „Biographischen Lexikons des Landes Österreich ob der Enns" (Krackowizer - Berger 1931) hervor. Krackowizer war, wie es in einem Nachruf heißt, „seiner Begabung nach nicht Forscher, sondern eine ausgesprochene Sammlernatur", das liege in seiner Familie begründet, sein Beruf als Archivar habe diese Anlage noch verstärkt (Zibermayr 1935: 95).

$\mathrm{Zu}$ seinem Karlsbad-Aufenthalt ist neben einem Reisetagebuch ${ }^{1}$ ein Erinnerungsalbum ${ }^{2}$ erhalten, in dem er - und das macht seinen oben erwähnten Hang zum exzessiven Sammeln deutlich - Belege aller Art vereint hat: Bildpostkarten, Konzertzettel, Fahrkarten, einen Stadtplan, eine Speisekarte, den Auszug der Karlsbader Kurliste mit der Nennung seines Namens, den Ordinationsbericht des behandelnden Arztes usw. Das Erinnerungsalbum wie das Reisetagebuch liefern vielfältige Ansätze zur Erforschung des westböhmischen Bädertourismus. Anhand dieser Quellen kann man Krackowizers Badereise detailliert rekonstruieren und so die Abläufe einer bürgerlichen Badereise ebenso nachvollziehen wie verschiedene Elemente der Alltagskultur des westböhmischen Kurortes Karlsbad um die Jahrhundertwende. Schließlich legen die benutzten Quellen auch Zeugnis ab vom Ausstellungswert, den der Kurgast Krackowizer dieser Reise beimaß, sie belegen den „Gebrauchswert [der Reise] für die Zeit nach der Rückkehr“ (Bausinger 1991: 350).

1 Reisetagebuch Karlsbad 1909, Oberösterreichisches Landesarchiv Linz, Nachlass Krackowizer, Tagebücher, Reisetagebücher, Druckwerke, 1861-1957, Nr. 2.

2 Das Erinnerungsalbum konnte von der Autorin antiquarisch erworben werden und ist heute im Besitz des Sudetendeutschen Museums München, Inv. Nr. 3809. 


\section{Die Quellen}

Im Nachlass Ferdinand Krackowizers im Oberösterreichischen Landesarchiv Linz befinden sich 24 Reisetagebücher, deren zeitlich erstes einer Holland/Belgien-Reise des Jahres 1869 und deren letztes einer Reise nach Kirchdorf im Jahre 1921 zuzuordnen ist. Neben dem der Badereise 1909 sind Tagebücher für Kuraufenthalte in Hofgastein in den Jahren 1906, 1907 und 1908 und Badgastein in den Jahren 1912 und 1914 vorhanden. ${ }^{3}$

Während Krackowizer für den Kuraufenthalt in Karlsbad ein Tagebuch, das sich auf schriftliche Einträge beschränkt, und das oben beschriebene Erinnerungsalbum, das - vollkommen unkommentiert - Belege der Reise zusammenfasst, angelegt hat, existieren für andere Reisen entweder nur die Tagebücher oder kombinierte Alben mit Text und (Bild-)Belegen. Bereits das als antiquarischer Zufallsfund zugänglich gewordene Erinnerungsalbum machte ein Nachvollziehen des Karlsbad-Aufenthaltes Krackowizers möglich. Die in einem zweiten Schritt unternommene Sichtung des Reisetagebuchs lässt nun in die Analyse auch die Sichtweisen und Gewichtungen des Reisenden selbst einfließen, seine Netzwerke rekonstruieren und Aspekte der kulturellen Praxis Badereise noch genauer herausarbeiten.

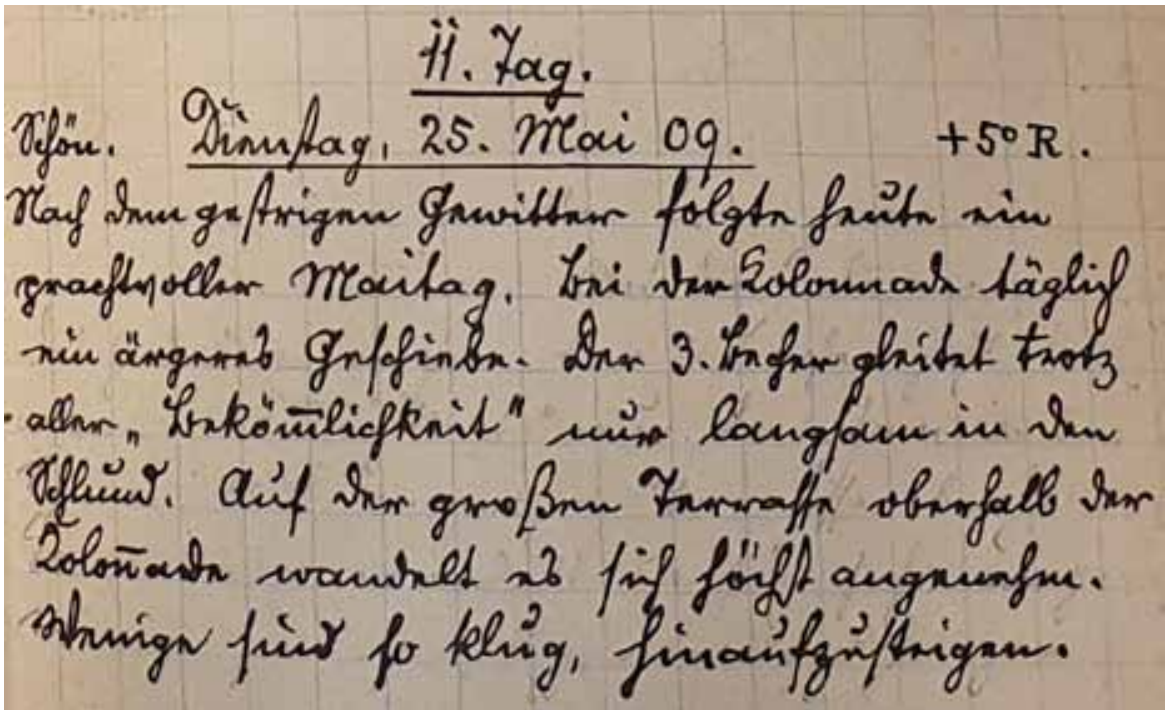

Abb. 1 Reisetagebuch Dr. Ferdinand Krackowizer, Karlsbad 1909, Detail aus S. 37.

3 Oberösterreichisches Landesarchiv, Nachlass Krackowizer, Tagebücher, Reisetagebücher, Druckwerke, 1861-1957, Nr. 2. 
Die schriftlichen Aufzeichnungen Krackowizers folgen wie die meisten Tagebücher dem diaristischen Prinzip der Tagestaktung (Holm 2008: 26) (Abb. 1). Während der Hauptteil des Textes, den Regeln eines Reisetagebuchs entsprechend, durch den Aufbruch nach Karlsbad und die Rückkehr aus Karlsbad begrenzt ist, hat Krackowizer zusätzlich eine $\mathrm{Zu}$ sammenstellung der Kosten eingefügt und - so ist anzunehmen - vor der Reise eine Liste der Personen eingetragen, die er mit Postkarten aus Karlsbad bedenken möchte, und diese dann jeweils mit einem Strich für eine geschriebene Karte versehen. Zudem sind einige Notizen zu finden, die auf dem Autor interessant erscheinende Besonderheiten von Karlsbad verweisen und - stehen sie doch getrennt vom eigentlichen Text - evtl. ebenfalls bereits vor der Reise notiert wurden. Sie sind als vor Ort nachzuprüfende kulturhistorische Notizen zu werten.

Die immer nach demselben Muster vorgenommenen Tagebucheinträge zeichnen das Bild eines systematisch arbeitenden, disziplinierten Menschen. Jeder Tag der Reise wurde nummeriert und mit Datum, Angaben zum Wetter im Allgemeinen und zur Temperatur begonnen.

Dass Krackowizer die Dokumentation seiner Reise ernst nahm, belegt etwa folgende Äußerung vom 29. Mai 1909: „Das Führen des Tagebuchs, sowie die Beantwortung der Briefe nehmen das Schläfchen nach Tisch weg" (Reisetagebuch: 51). Krackowizer beschrieb das Führen des Tagebuchs jedoch nicht als lästige Pflicht, sondern als beruhigende Bereicherung der Reise. So heißt es im Eintrag vom 16. Mai 1909: „Ich ruhe im stillen Hinterzimmer aus und schreibe bei dem Gesange einer fleißigen Amsel in diesem Tagebuche" (Reisetagebuch: 5). Und am letzten Karlsbad-Tag vermerkte er: „Nochmals wird der feine Terlaner mit dem erfrischenden Gießhübler ${ }^{5}$ getrunken und zum letztenmal dieses Tagebuch beschrieben. Es waren mir stets liebe Stunden einsamer Stille und Sammlung des Geistes" (Reisetagebuch: 70). Der Schreiber sah in dem Tagebuch also auch ein Mittel der Selbstdisziplinierung, wie es etwa Günter Oesterle für Reiseaufzeichnungen adeliger Teilnehmer von Kavalierstouren des 18. Jahrhunderts beschrieben hat. Auch die Rechenschaft über das ausgegebene Geld, die „Abwägung von Geld- und Zeitnutzung“" weist in diesen Zusammenhang. „Fiskalische wie moralische Abrechnung“ fanden im Reisetagebuch Platz (Oesterle 2008: 101).$^{6}$

4 Das Bild der fleißigen Amsel wurde hier, so ist anzunehmen, eingefügt, um damit auf den eigenen Fleiß zu verweisen.

5 Als Abend- bzw. Nachttrunk nahm Krackowizer stets Wein aus Terlan (Südtirol) und Mineralwasser aus Gießhübl/Kyselka zu sich.

6 So notierte Krackowizer etwa jede noch so kleine Abweichung von dem durch den Arzt festgelegten Speiseplan in seinem Tagebuch. 


\section{Die Badereise als Form des frühen Tourismus}

Die moderne Badereise entstand im 18. Jahrhundert und hatte sich im 19. Jahrhundert zum "frühtouristischen Standardprodukt" entwickelt (Kos 1991b: 220). Neben dem Wunsch nach Gesundung waren es Motive wie Unterhaltung, Geselligkeit und die vielfältigen Möglichkeiten des Knüpfens und Pflegens von Kontakten, die "Jung und Alt, Gesund und Nichtgesund" (Stürmer 1990: 30) in die Bäder führten. Dabei war es häufig wichtiger, „wo man trank [...], als was man trank“ (Stürmer 1990: 30).

Die Reise ins Bad, ursprünglich ein Privileg adeliger Schichten, entwickelte sich seit dem frühen 19. Jahrhundert zu einem Statussymbol des aufsteigenden Bürgertums. Das Streben nach Gesundheit legitimierte diese Reise: „Das Badeleben ist beschäftigter und pflichtgemäßer Müßiggang, der einzige also, der keinerlei Anlaß zu Selbstvorwürfen gibt. Man hat eigentlich nichts zu tun und erübrigt trotzdem keine freie Zeit" (Hanslick 1987: 345). „Wenn es mir einmal so gut gehen wird, daß es mir schlecht gehen darf, will ich auch hinfahren" (Polgar 1928: 215) - mit diesen Worten über Karlsbad beendete Alfred Polgar seinen Essay „Städte, die ich nicht erreichte" aus dem Jahre 1927. Karlsbad stellte, das macht nicht nur dieses Zitat deutlich, einen großbürgerlichen Sehnsuchtsort dar.

Auf der einen Seite blieben die Quellen, die bereits früh von der an Bedeutung gewinnenden Balneologie als medizinisch wirksam anerkannt waren, bis zur Einführung einer Sozialversicherung um die Jahrhundertwende „elegante Enklaven“ (Stürmer 1990: 30), „mondäne Orte einer vornehmen Gesellschaft" (Fuhs 1992), auf der anderen Seite waren die Bäder für bestimmte gesellschaftliche Gruppen, wie die erstmals ohne Begleitung reisenden Frauen (Lorenz 1949: 251), emanzipatorische Orte.

Weil im Bad alles auf Vitalität und Lebensfreude hinweisen sollte (Kos 1991b: 221), waren ernsthaft kranke Gäste in Sanatorien am Rande der Badeorte untergebracht, während sich in den Zentren und an den gesellschaftlichen Treffpunkten zum großen Teil „Titularkranke“ aufhielten, wie es in zeitgenössischen Quellen ${ }^{7}$ heißt. Wolfgang Kos hat beschrieben, wie auf der „sozialen Experimentierbühne“ (Kos 1991a) Kurbad, an einem - so Sebestyén - „durch und durch unnatürlichen Ort“ (Sebestyén 1980: 36ff.), die Fähigkeit, eine „Doppelexistenz zwischen intimer Wahrheit und sozialer Kostümierung“ (Kos 1991a: 43f.) zu führen, perfektioniert wurde. Obwohl das Bad immer wieder als egalitärer Ort beschrieben wurde und der Kuraufenthalt als Zeitspanne galt, in der es möglich war, soziale und regionale Bindungen zu überschreiten, standen, so Wolfgang

7 Etwa bei [Kretschmann] 1798: 176: „Viele der Gäste sind bloß titular [...].“ 
Kos, „ständische Annäherung und schroffe Gesten der Abschließung [...] oft hart und unvermittelt nebeneinander" (Kos 1991a: 48).

\section{Zur Kur nach Karlsbad}

Der Sage nach entdeckte Kaiser Karl IV. die warmen Quellen von Karlsbad auf einer Hirschjagd im Jahre 1347. Als Entdecker der eigentlichen Heilkräfte des Karlsbader Wassers gilt der Arzt Wenzel Payer: In einem medizinischen Traktat empfahl er 1522 das Trinken der heißen Quellen und leitete so die allmähliche Ablösung der Badekur durch die Trinkkur ein. Die erste physikalisch-chemische Untersuchung der Quellen lieferte Dr. David Becher (1725-1792). Ihm sind auch die Verbesserung der Kurmethoden, Vorschläge zu einem effizienteren Gebrauch der Quellen und eine architektonische Ausgestaltung des Kurortes zu verdanken (Burachovič 2000: 61-65).

Der seit dem 18. Jahrhundert stark aufgeblühte Karlsbader Kurbetrieb wurde durch einen Ausbau der balneologischen Forschung professionalisiert und weiträumig propagiert. Einen großen Anstieg der Gästezahlen brachte der Anschluss an das europäische Eisenbahnnetz im Jahr 1870. Karlsbad lag zu seiner Blütezeit - so Triendl-Zadoff - „an den Hauptverkehrsverbindungen zwischen West- und Osteuropa“, u.a. passierte der Orientexpress Oostende-Istanbul die Stadt (Triendl-Zadoff 2007b: 293). Hatte man im Jahr 1850 noch 6.638 Kurgäste gezählt, waren es 1871 bereits 17.974, 190151.454 (Oertl - Ludwig - Becher 1902: 201), 190559.736 und 190966.153 (Karlsbader Kurliste 1910: Umschlag-Rückseite).

Während 1860 noch eine Kurdauer von 4-5 Wochen üblich war, blieben die Gäste 1861 bis 1901 durchschnittlich 28 Tage und danach 3-4 Wochen (Leeb 1902: 613). 1901 kamen auf 1.000 männliche 924 weibliche Kurgäste (Leeb 1902: 624). Unter den 51.454 Kurgästen des Jahres 1901 waren 16.199 Gäste aus dem Bereich Handel, 4.936 Rentiers und Hausbesitzer, 4.588 Gewerbetreibende und 4.330 Beamte (Leeb 1902: 626). Während 1901 19.539 Kurgäste aus Preußen stammten, kamen 5.743 aus Russland, 5.504 aus „Österreich unter der Enns“, 4.692 aus Böhmen, 3.040 aus Galizien und Lodomerien, 2.247 aus Amerika und nur 151 aus Österreich ob der Enns (Leeb 1902: 628).

Nach der Volkszählung vom 31.12.1900 besaß der Stadtbezirk Karlsbad 14.637 Einwohner, davon waren 6.518 männlich, 8.119 weiblich. Der katholischen Konfession gehörten 12.735 Personen an, der evangelischen 466 , der „israelitischen“ 1.405 , unter „andere“ [Konfession] sind 31 Personen angegeben. Umgangssprache von 14.006 Personen war deutsch, von 129 Personen „böhm., mähr., slowakisch“ und fünf Personen bedienten 
sich einer anderen Umgangssprache (K.K. Statistische Zentralkommission 1905: 290). Nach der Volkszählung vom 14. April 1920 lebten im Bezirk Karlsbad 72.471 Deutsche, 1.811 Tschechen, 167 Juden, 63 übrige und 2.363 „Ausländer“, davon gaben 71.871 ihre Konfession als römischkatholisch, 1.828 als evangelisch, 2.681 als jüdisch an (Statistický lexikon 1923: 256).

„Ein fashionabler Aufenthalt war Karlsbad schon in der zweiten Hälfte des 18. Jahrhunderts“, heißt es 1908 in dem Handbuch „Österreichs Reiseländer“. Und weiter lesen wir: „Karlsbad ist der bevorzugte Sitz des Wohllebens und der Eleganz geblieben bis auf den heutigen Tag" (Horst 1908: 361). Im Gedenkbuch der Bezirkshauptmannschaft Karlsbad lesen wir zum Jahr 1909, die Zahl der Kurgäste habe zugenommen, „leider in der Qualität nicht". ${ }^{8}$

Die steigende Zahl der Kurgäste bedingte auch neue Ansprüche an die Architektur der Bäder.

"Karlsbad ist nicht nur das Mekka für Hunderttausende von Kranken [...], sondern auch ein herrlicher Sommeraufenthalt für Gesunde, mit dem großen Vorzug, verfeinerten Lebensansprüchen in Wohnung, Nahrung und Körperpflege zu genügen." (Exner 1929: 244)

\section{Ferdinand Krackowizers Reise nach Karlsbad}

Krackowizer kam am 15. Mai 1909 abends in Karlsbad an. Er nahm Wohnung bei Carl Zörkendörfer im "Logirhaus Insel Malta“ (Abb. 2) in der Kaiserstraße 73 (Adreßbuch 1906/07: 132). ${ }^{9}$ Mit der Privatiere Johanna Dimmel war bei seiner Ankunft bereits ein Kurgast aus Linz dort einquartiert. ${ }^{10}$ Die anderen Zimmer waren - liest man die Karlsbader Kurlisten ${ }^{11}$ vom April und Mai 1909 - belegt von Badereisenden aus Nürnberg, Dresden, Danzig, Berlin, Stuhlweissenburg etc.

8 Gedenkbuch der Bezirkshauptmannschaft Karlsbad/Kronika okresního hejtmanství, 1888-1927, Státní okresní archiv Karlovy Vary, S. 193.

9 Zörkendörfer war Sparkassenbeamter und Eigentümer des Hauses „Insel Malta".

10 Karlsbader Kurliste 1909, Nr. 36, 1. Mai 1909, unter Nr. 2356: „Frau Privatiere Johanna Dimmel, Linz, Insel Malta, Kaiserstrasse“.

11 Seit dem Jahr 1795 liegen für Karlsbad gedruckte Kurlisten vor, in denen Name, Herkunft und Unterkunft aller Kurgäste verzeichnet wurden. Mit Beschluss desselben Jahres wurde 1796 das bisher als freiwillige Spende laufende Badegeld durch die Badegeldpflicht ersetzt. Dazu Augustin 2010: 41-42. 


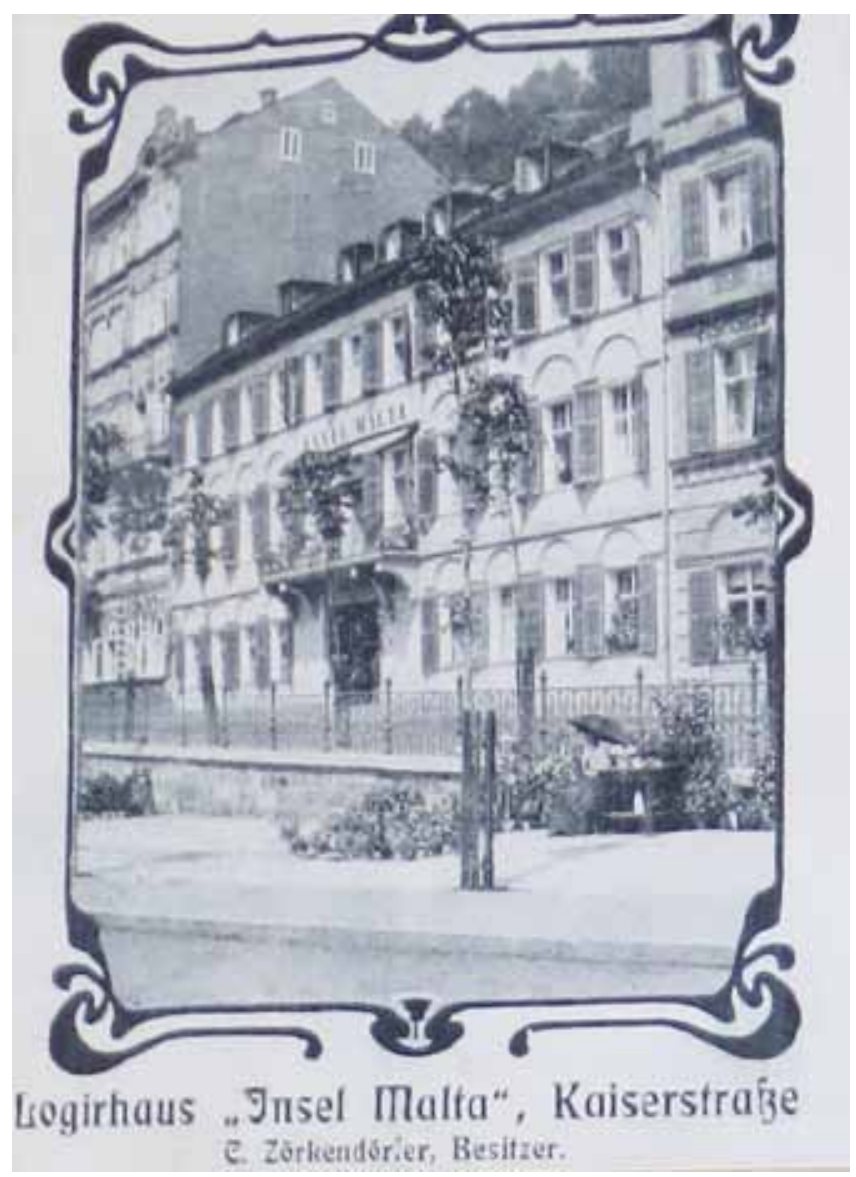

Abb. 2 Logierhaus „Insel Malta“, „Carte de Visite“ im Erinnerungsalbum Krackowizers.

Am 16. Mai 1909 fand Krackowizers Eingangsuntersuchung bei dem Kurarzt Dr. Josef [Joseph] Ruff (Schwarzes Ross, Mühlbrunnstraße) statt. ${ }^{12}$ Ruff, der 1864 in Verböcz (Komitat Ugocsa/Ugotsch/Ugocsa vármegye) in Ungarn geboren wurde und 1912 in Karlsbad starb, galt als Spezialist für die Behandlung von Stoffwechselkrankheiten. Nach Stationen in Wien, Stuttgart und Brünn war er nach Karlsbad gelangt, wo er 1906/1907 als Obmann des Karlsbader Ärzte Vereines (Adreßbuch 1906/07: XLIX) aufgeführt ist. Er trat als Autor verschiedener populärmedizinischer Werke hervor (Ruff 1884; Ruff 1887; Ruff 1889; Ruff 1894) und war auch regionalhistorisch und literarisch tätig. So war er etwa Vorstandsmitglied des

12 Laut Gintl hielt Ruff „morgens 7-9“ und „nachmittags 3-5“ Sprechstunden ab. Gintl (1908): 96. 
1900 gegründeten „Journalisten- und Schriftsteller-Vereins Concordia“ in Karlsbad. ${ }^{13} 1899$ hatte er den Gäste-Führer „Karlsbad wie es war und wie es ist" (Ruff 1899) herausgegeben, der in mehreren Auflagen erschien und sich - wie eine Notiz im Reisetagebuch verrät - auch im Besitz Krackowizers befand. Als Präsident der „Stuttgarter österreichisch-ungarischen Colonie“, des Vereines von Oesterreichern in Stuttgart, ${ }^{14}$ erhielt Ruff 1880 das Ritterkreuz des Franz-Joseph-Ordens ${ }^{15}$ verliehen. Die Tatsache, dass der Arzt jüdischen Glaubens war, wird von Krackowizer nicht erwähnt. Allerdings spielt er auf gemeinsame Bekannte an, wenn er Ruff „einen Freund der Kaser in Stuttgart" nennt (Reisetagebuch: 4).

Ruff verordnete seinem Patienten eine Diät (Abb. 3), ${ }^{16}$ die erahnen lässt, dass dieser unter Leber- oder Gallenproblemen litt, eine Vermutung, die durch eine Notiz im Reisetagebuch bestätigt wird (Reisetagebuch: 4). Empfohlen wurden 2 Glas Mühlbrunnen (49,7 Grad Celsius) und 1 Glas Bernhardbrunnen (66 Grad Celsius) pro Tag, ${ }^{17}$ als „sonstige[s] Kurmittel“ dreimal wöchentlich Moorumschlag..$^{18}$ Bei der dritten Konsultation am 27. Mai 1909 beschied der Arzt ein Ende des Kuraufenthaltes nach 21 Tagen (Reisetagebuch: 45).

Für die Kur gab Krackowizer insgesamt 480 Kronen aus (Reisetagebuch: 72), eine Summe, die im Reisetagebuch penibel aufgedröselt wurde.

Aus dem Reisetagebuch lässt sich der für eine Kur übliche stark reglementierte Tagesablauf Krackowizers ablesen. Das Immer-Gleiche des den Kurgast in ein enges Korsett zwingenden Programmes wurde ab dem frühen 20. Jh. häufig zum Thema auf Bildpostkarten gemacht. Eine im Erinnerungsalbum überlieferte Bildpostkarte mit Sprudelstein-IntarsienRahmen $^{19}$ (Abb. 4) beschreibt die alltägliche Kurroutine.

13 Vereinsnachrichten. Verein Concordia, in: Pilsner Tagblatt, I. Jg., Nr. 59 (7. Juni 1900), S. 3.

14 Ein Verein von Oesterreichern in Stuttgart, in: Salzburger Volksblatt, 19.11.1874, S. 4.

15 Notiz „Allerhöchste Erschließungen“, in: Wiener Zeitung Nr. 127 (5.6.1880), S. 4.

16 Als empfohlene Diät notierte der Arzt: „Frühstück: Verkehrter mit Wassergebäck 1-2 weiche Eier oder Schinken. Mittagessen: Suppe, Fisch (blau gesotten) Braten (nicht panirt [sic]) grünes Gemüse oder frisch gekochtes Obst 1/8 Wein mit Gießhübler. Nachmittags: 1 kl. Kapuziner (hell) Abends: Braten oder kalter Aufschnitt." Erinnerungsalbum 1909, unpaginiert.

17 Zum Bernhardsbrunnen Fleckles 1838: 285.

18 Ordinationszettel des Dr. Josef Ruff, in: Erinnerungsalbum 1909, unpaginiert.

19 Das stark mineralienhaltige Karlsbader Wasser führt zu Ablagerungen, die herausgebrochen, geschliffen und zu Intarsien für verschiedene Andenkenobjekte wie Kassetten, Taschenmesser, Brillenetuis etc. verarbeitet wurden. Die L. V. Endersche Kunstanstalt in Neutitschein/Nový Jičín stellte um die Jahrhundertwende Karten mit Sprudelstein-Dekor her. Dazu: Grundmann/Zechner 2004. 


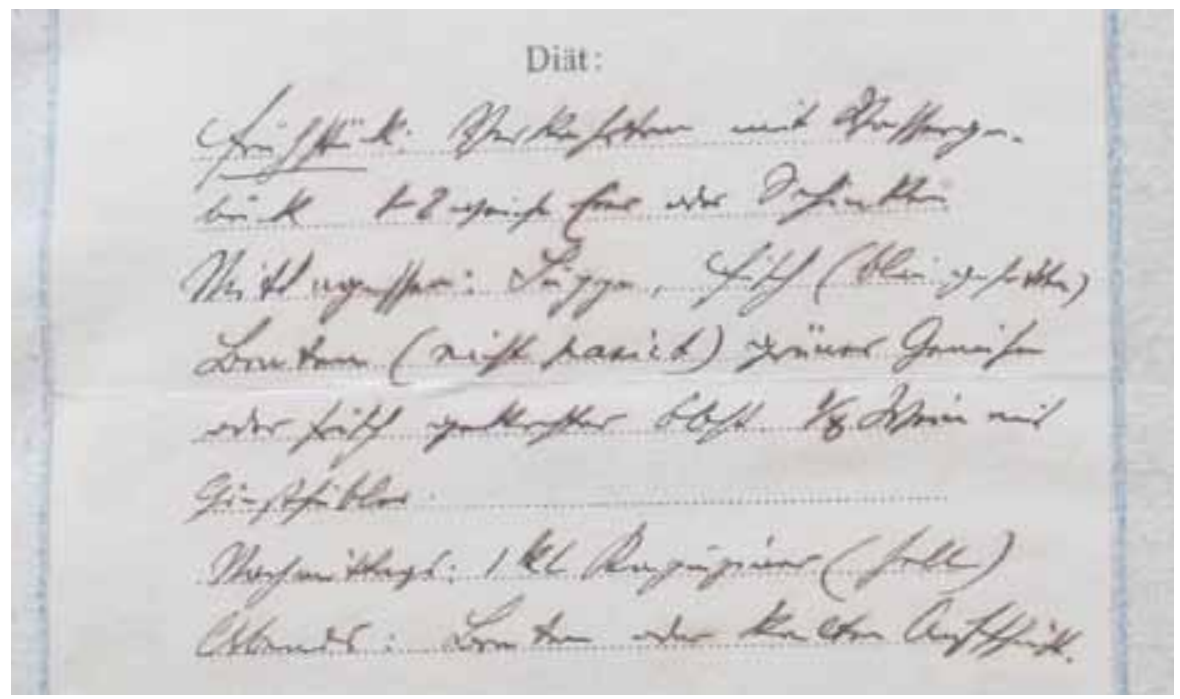

Abb. 3 Detail aus dem Ordinationsbericht Dr. Ruffs, Erinnerungsalbum des Ferdinand Krackowizer.

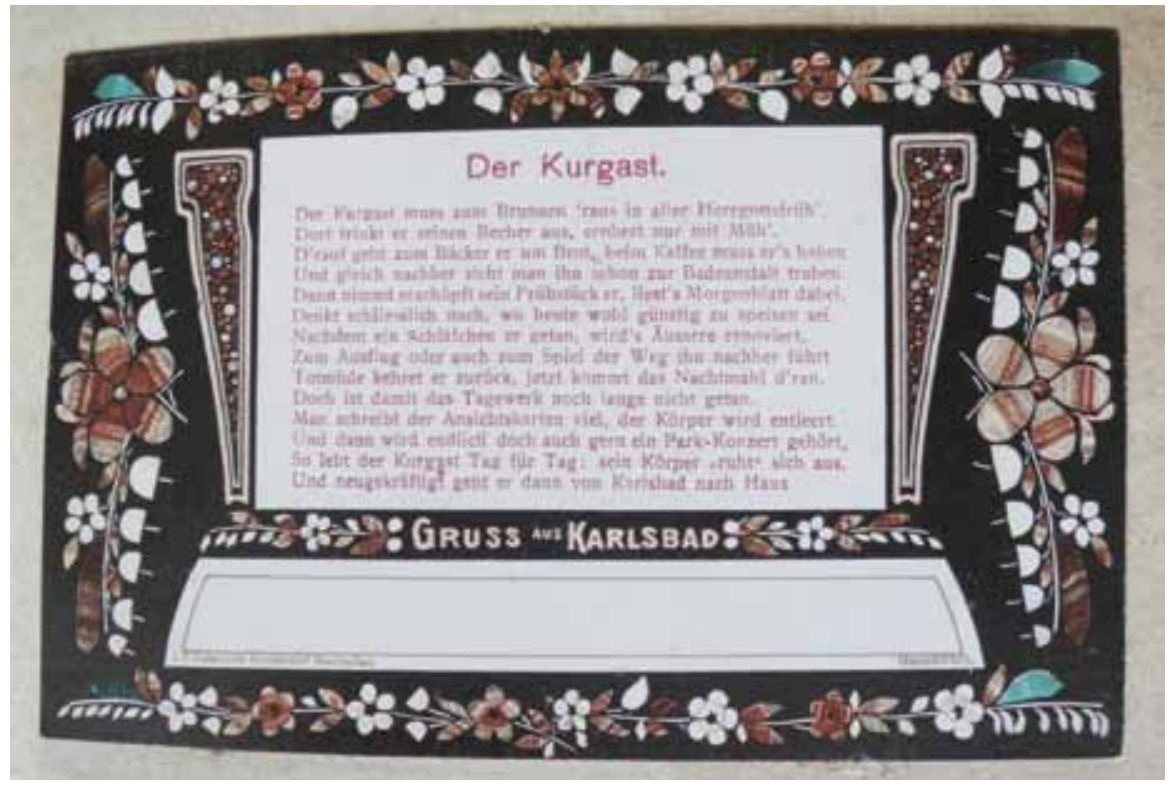

Abb. 4 Postkarte mit Sprudelstein-Dekor aus dem Erinnerungsalbum Krackowizers, Neutitschein/Nový Jičín, frühes 20. Jh. 


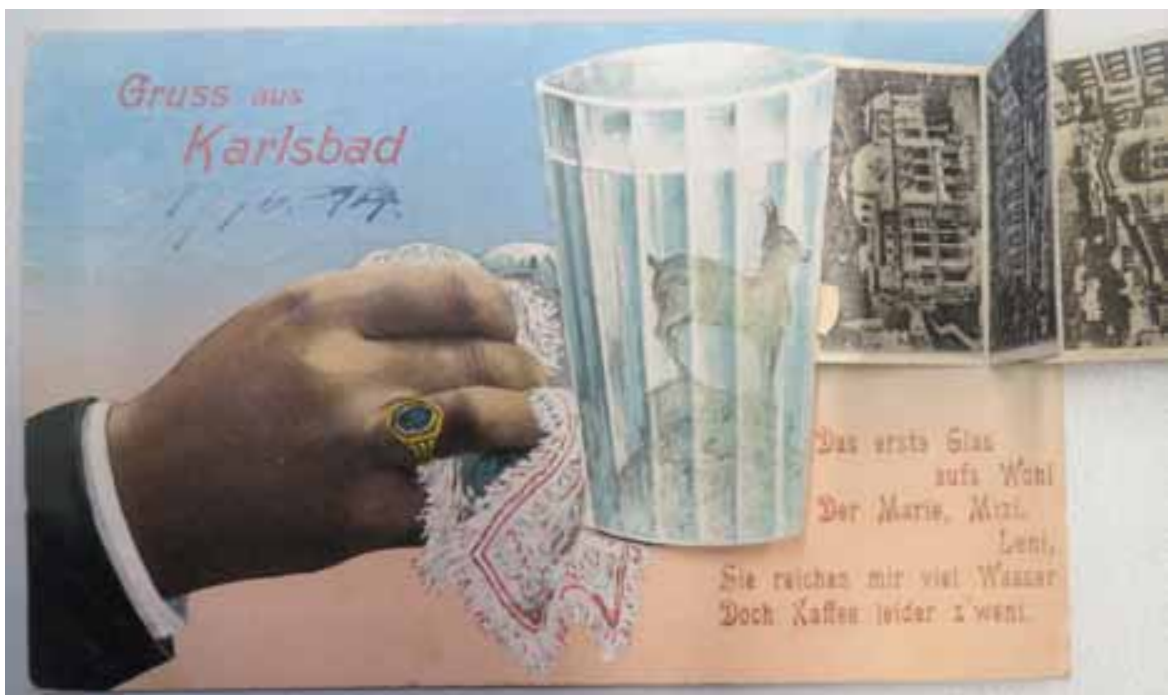

Abb. 5 Leporellokarte „Gruss aus Karlsbad“, ohne Verlagsangabe, gelaufen 1914: Eine beringte Männerhand hält Badebecher und dazugehöriges Tüchlein. Im Text wird auf den angeratenen Verzicht von zu viel Kaffee hingewiesen.

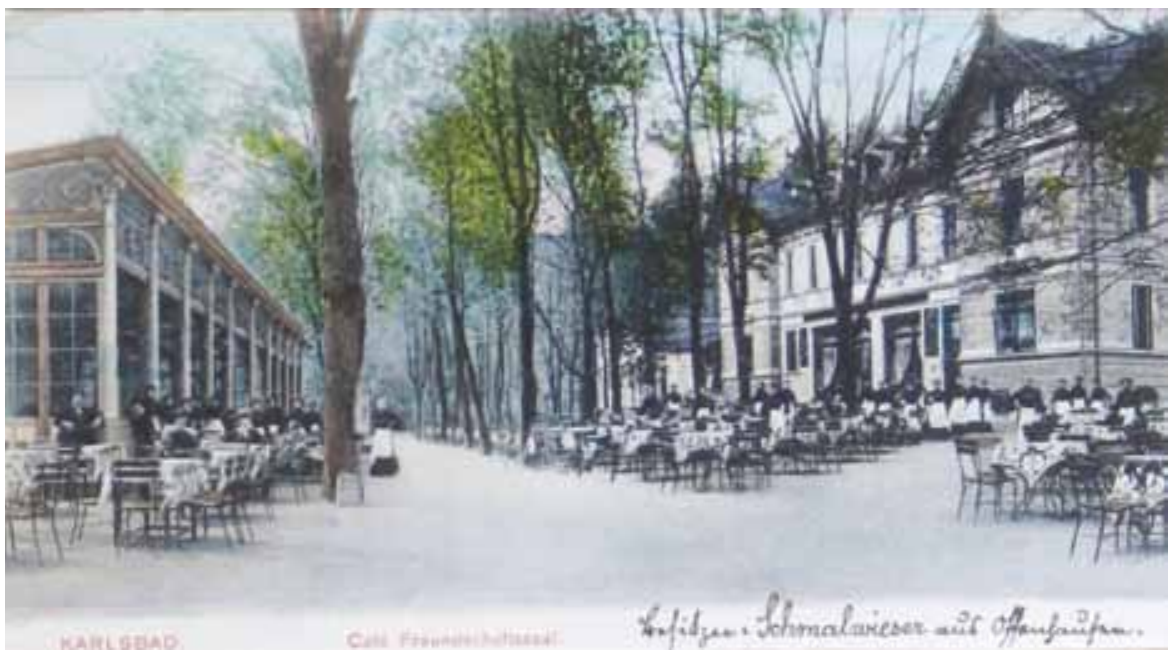

Abb. 6 Bildpostkarte des Café Freundschaftssaal, handschriftlicher Zusatz: „Besitzer: [Martin] Schmalwieser aus Offenhausen“, Erinnerungsalbum Krackowizers 1909. 
Am ersten Tag der Kur erstand Krackowizer einen Trinkbecher „sammt Tüchlein und Riemen um 1 k 90 h" (Reisetagebuch: 3) (Abb. 5). Von nun an war er jeden Morgen zwischen 6:00 Uhr und 6:30 Uhr an den Quellen, um die verordneten drei Becher „schrittweise trippelnd im Haufen“ (Reisetagebuch: 3) zu trinken. Die Kurkapelle begleitete diese Morgentrinkstunde, an die sich ein längerer Spaziergang zu einem der in der Umgebung liegenden Frühstückslokale, den „Mokka-Tempeln“ (Reisetagebuch: 3), wie er sie nannte, anschloss. Wie die Belege im Erinnerungsalbum und die Einträge im Reisetagebuch zeigen, wechselte Krackowizer zwischen verschiedenen Cafés ab. Mit Abstand am häufigsten war er jedoch Gast im „Freundschaftssaal“, einem Kaffeegarten, mit oberösterreichischem Pächter, wie er auf einer Bildpostkarte vermerkte (Abb. 6).

Auf das Frühstück, das immer wieder als der „größte Hochgenuß aller Tafelfreuden in Karlsbad" (Kronser 1861: 96) beschrieben wird, folgten ein längerer Spaziergang oder Anwendungen im Kurhaus. Alle Restaurants in Karlsbad boten kurgemäße Diätküche an, dennoch nahm Krackowizer - mit zwei Ausnahmen - täglich im selben Restaurant, dem „Morgenstern“, sein Mittagessen ein. Er notierte jede Mahlzeit mit dem jeweiligen Preis und dem gegebenen Trinkgeld. So schreibt er etwa am 21. Mai 1909: „Mittagstafel im Morgenstern: Griesnokensuppe d.h. in gehaltloser warmer Brühe ein [im Original unterstrichen] Nokerl. Karotten mit faschierter Kotelette. Aepfelpürée. (70 h.)“ (Reisetagebuch: 25). Nach dem Mittagessen, dessen Beschreibung Krackowizer häufig Anlass dazu bot, über die einzuhaltende Diät zu klagen, folgte ein kurzer Mittagsschlaf und danach ein Ausflug, eine Wanderung oder ein Museumsbesuch. Am späteren Nachmittag oder am Abend besuchte Krackowizer Konzerte (Abb. 7) oder verbrachte einen ruhigen Abend in seiner Unterkunft und las die aus der Heimat nachgesendete Linzer Tages-Post.

\section{Krackowizers Blick auf Karlsbad}

\section{Der Ort}

Vom ersten Tag seines Karlsbad-Aufenthaltes an lobt Krackowizer die Sauberkeit, die Freundlichkeit, die gute Organisation des Ortes: „Wohltuend ist die außerordentliche Reinlichkeit [im Original unterstrichen] auf allen Plätzen, Strassen und Wegen. Gereinigt werden diese nur bei Nacht. Ebenso wird während der Badezeit nirgends gebaut. Stramme, deutsche, höfliche, städtische Schutzleute, mit einem Waffenröcke [sic] ähnlich den Gendarmen und mit einer Pickelhaube, handhaben die Ordnung." (Reisetagebuch: 14) Die der Stadt zugeschriebenen Tugenden werden dabei 


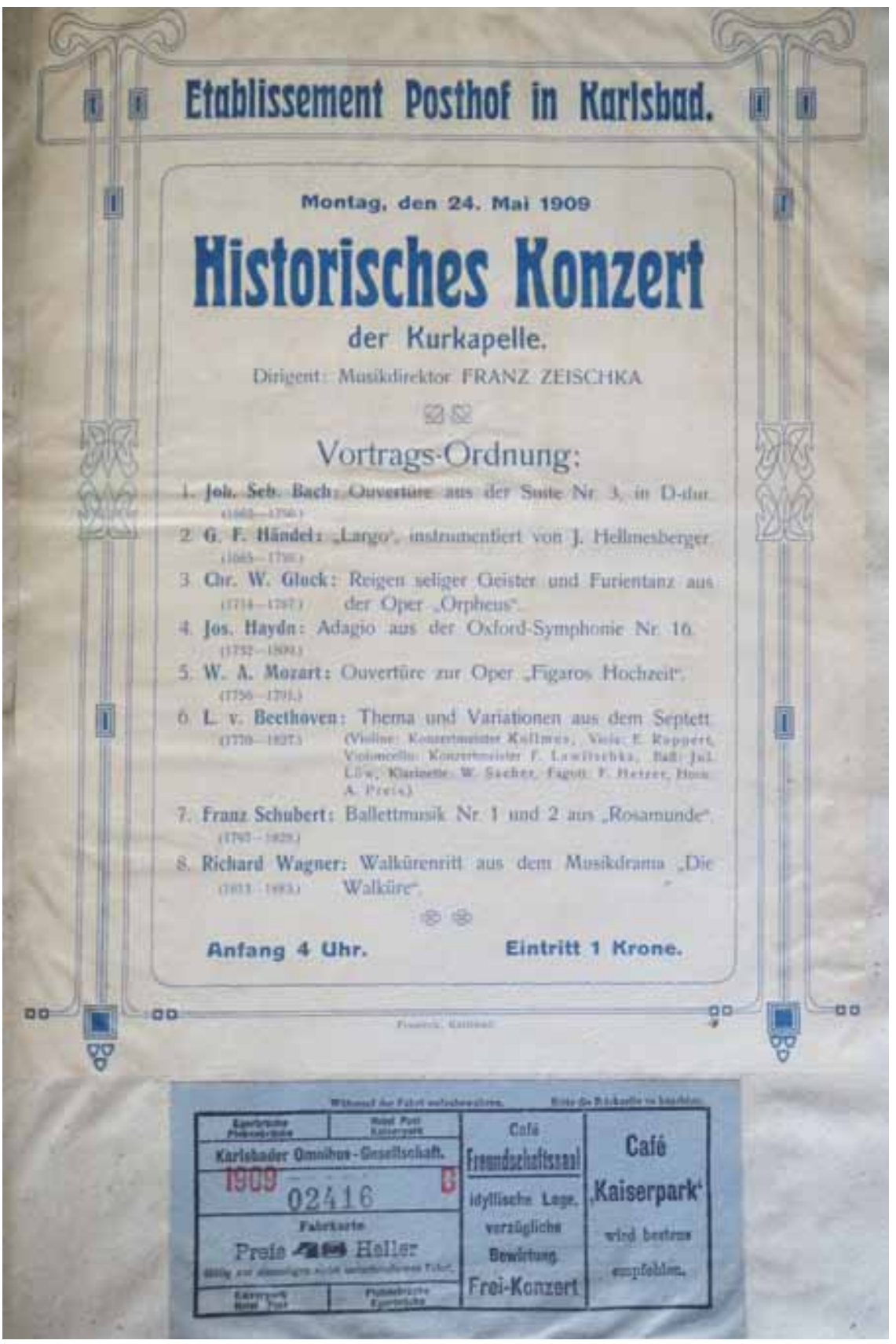

Abb. 7 Seite aus dem Erinnerungsalbum Krackowizers mit Programmzettel eines Konzertes im Posthof und Omnibuskarte mit Hinweis auf ein Freikonzert im Café Freundschaftssaal. 
immer als deutsche Tugenden beschrieben: „Eine musterhafte Stadtverwaltung zeigt überall ihr Wirken. Alles kerndeutsch." (Reisetagebuch: 5) An mehreren Stellen beschreibt Krackowizer Karlsbad als mögliches Vorbild für andere Städte. So berichtet er etwa von den Bürgersteigen der steilen Straßen, sie seien „kräftig gerippt, so daß man nicht leicht ausgleitet" und beendet diese Beobachtung mit einem "Nachahmenswert." (Reisetagebuch: 18) Alleine Teile des Kurpublikums und die in Karlsbad vorgefundenen Preise sind Thema seiner Kritik.

\section{Das Publikum}

Die Internationalität Karlsbads scheint Krackowizer zumeist als Bereicherung aufzunehmen, wie folgende drei Beispiele zeigen: „Wir kamen auch zum Hirschensprung und hinüber in das elegante Stadtviertel Westend, wo uns ein Mohr und ein Indier [sic] mit weißem Turban begegnet", heißt es etwa in seinem Reisetagebuch (Reisetagebuch: 48) und an anderer Stelle lesen wir dort: „Auf dem Wege kaufte ich bei fliegenden Händlern von einem Japaner eine Steinschnitzerei seiner Inselheimat um $1 \mathrm{~K}$.“ (Reisetagebuch: 36) und „Der polnische Pfarrer war mein Tischgenosse, mit dem ich durch lateinische Brocken den internationalen Verkehr vermittelte. So bringt fast jeder Tag Neues!" (Reisetagebuch: 54)

Neben dem interessierten, freundlichen Beschreiben von Angehörigen fremder Ethnien gibt es jedoch eine Gruppe von Kurgästen, denen von Seiten Krackowizers heftige Ablehnung entgegenschlägt. Es sind die jüdischen Kurgäste, die sein Missfallen erregen: „Unter dem internationalen Publikum wandelte hoheitsvoll ein Mann in langem violetten Talare aus Sammt [sic], einen steifen Halbzilinder [sic] auf dem Kopf u. Schnittlauchlocken. Polnische Juden, mehr als genug." (Reisetagebuch: 10) Dabei stört er sich vor allem an Äußerlichkeiten: „Die Judenfrauen tragen greuliche Perücken“ kommentiert er etwa einen Verhaltenskodex orthodoxer Juden. Auch die Charakterisierung „,von hebräischem Urstamm“ (Reisetagebuch: 39) ist abwertend zu interpretieren.

Die jüdischen Kurgäste werden von ihm meist als reiche Emporkömmlinge beschrieben, mit denen er sich nicht gemein machen möchte: „Viel heiterer [als im Café Pupp, EF] ists im Freundschaftssaal. Dort viele lustige alte Männer; hier steife protzige Jüdinnen“ (Reisetagebuch: 42) schreibt er, oder „Im Omnibus fuhr eine spanische Jüdin mit, die mühsam mit mir Italienisch sprach und ihre Finger mit kostbaren Brillanten ganz steif hielt. Heißt das ä Grandezza [sic]“" (Reisetagebuch: 26).

In vielen europäischen Kurorten kam es Ende des 19. und zu Beginn des 20. Jahrhunderts zur offenen Diffamierung jüdischer Kurgäste. Frank Ba- 
johr hat das am Beispiel der deutschen Kurbäder gezeigt (Bajohr 2003). Anhand der vor allem in Karlsbad und Marienbad beliebten und sehr verbreiteten Judenspottkarten, von denen einige auch in Krackowizers Erinnerungsalbum zu finden sind, kann dies für die westböhmischen Bäder nachgewiesen werden. ${ }^{20}$ Ein Großteil dieser Karten stammt aus dem Verlag Ottmar Zieher in München. ${ }^{21}$ Käufer dafür waren - so Peter K. Klein - vor allem in der Mittelschicht des städtischen Bürgertums zu fin-

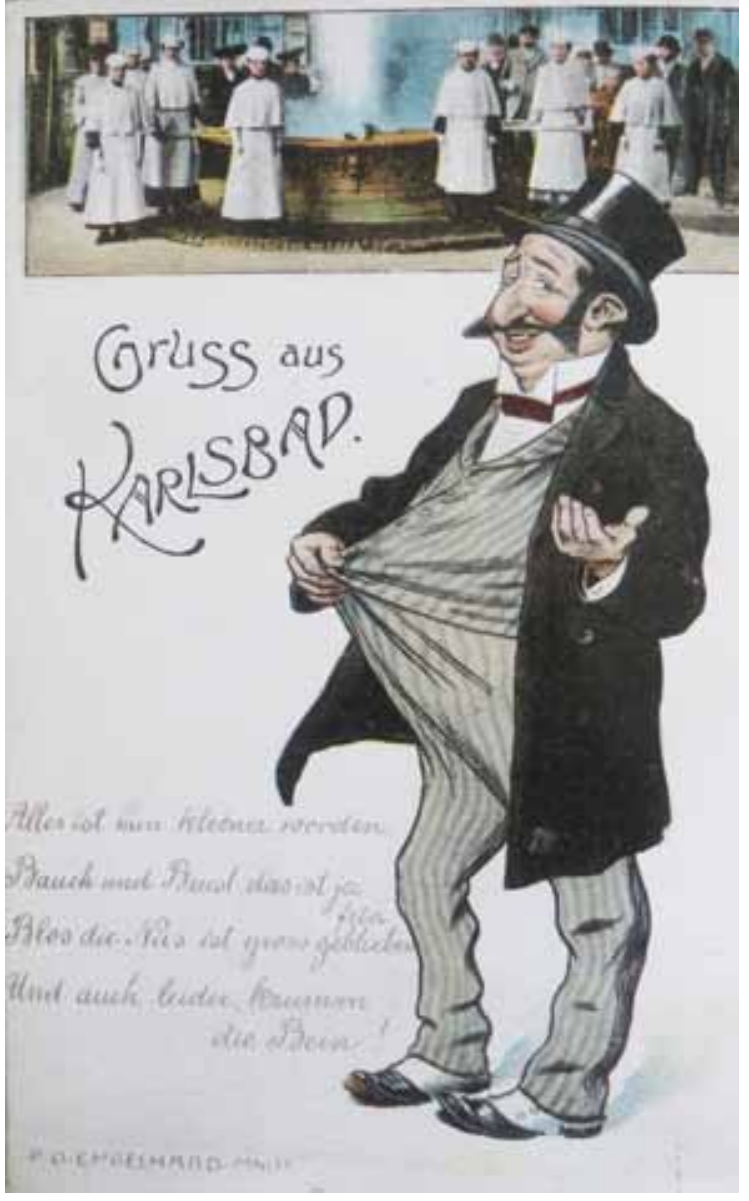

Abb. 8 Bildpostkarte „Gruss aus Karlsbad“, gestaltet von Paul Otto Engelhard, wohl Verlag Otmar Zieher, München, um 1905.

20 Die Blütezeit der Bildpostkarte liegt zwischen 1885, dem Jahr, in dem sie offiziell zugelassen wurde, und 1914 (Einführung der geteilten Rückseite 1905). Judenspottkarten nehmen innerhalb der um die Jahrhundertwende versendeten Bildpostkarten einen geringen Anteil von 1-3\% ein. Dazu: Klein 2006: 126.

21 Der Verlag war seit 1900 als lithographische Kunstanstalt registriert. Klein 2006: 127. Dazu auch: Triendl-Zadoff 2007a: 102. 
den (Klein 2006: 148). Auf den Karten erfolgte zunächst meist eine physiognomische Diffamierung der dargestellten jüdischen Kurgäste (Klein 2006: 139). Eine von Krackowizer gesammelte Karte, entworfen von dem Illustrator und Graphiker Paul Otto Engelhard, die auf den Erfolg der Diät während der Kur anspielt, macht das deutlich (Abb. 8). „Alles ist nun kleener worden: / Bauch und Brust das ist ja fein“ heißt es da, und weiter: „Blos die Nas ist groß geblieben / Und auch leider, krumm die Bein!" $\mathrm{Zu}$ sehen ist ein vornehm gekleideter jüdischer Kurgast, der durch das Präsentieren seiner jetzt zu weiten Weste den Abnehmerfolg beweisen möchte. ${ }^{22}$

Dass Krackowizer auf der einen Seite einen jüdischen Arzt konsultierte, auf der anderen Seite jedoch antisemitische Spottkarten kaufte und archivierte, in einem als antisemitisch bekannten Logirhaus wohnte und in einem ebensolchen Gasthaus aß - sowohl das Logierhaus „Insel Malta“ als auch das Restaurant "Morgenstern“ wurden vom Verein zur Abwehr des Antisemitismus als antisemitisch eingestuft (Antisemitische Bade-, Kur- und Erholungsorte 1909: 164) -, lässt aufhorchen und erinnert an das von Frank Bajohr für die böhmischen Bäder beschriebene Phänomen des ,Winter-Antisemitismus'. Des guten Verdienstes wegen war man, so Bajohr, hier selbst den Fremden gegenüber freundlich, die man aus weltanschaulichen Gründen verachtete.

Diese „Melange aus geheuchelter Freundlichkeit während der Sommersaison und brachialem Antisemitismus in der Winterzeit" (Bajohr 2003: 149) hat der jüdische Humorist Julius Stettenheim 1901 mit folgenden Worten beschrieben: „Von besonderer Komik ist der Antisemitismus in Karlsbad. [...] Im Sommer haben die Antisemiten mit höflichstem ,Küß d'Hand' die Juden übers Ohr gehauen, im Winter nehmen sie keine Hand vor den Mund [...] Dann ist ihnen nichts heilig als nur die Zucker-, Nieren- und Lebersteinkranken, die in einigen Tagen wieder mit vollen Taschen erscheinen, die sie bei hellem Tag sorgfältig überfallen. Dann wimmelt Karlsbad von Herren ,von Cohn` und in den Läden wird jeder

22 Es ist wahrscheinlich, dass auch diese Karte bei Ottmar Zieher in München produziert wurde, da Engelhard für diesen Verlag immer wieder tätig war. - Das Beispiel des Münchner Malers Friedrich August von Kaulbach (18501920) macht deutlich, dass es aber auch einen anderen Umgang mit den jüdischen Kurgästen gab. Kaulbach fertigte während seiner Karlsbad-Aufenthalte 1909 und 1913 mehrere Skizzen jüdischer Kurgäste aus dem osteuropäischen Raum, die sein „dokumentarisches Interesse“ an der anderen Kultur deutlich machen und keine abwertende Funktion besitzen. Dazu: Dittmar 1992: 267 und 401-405. 
baronisiert, der im Winter wenigstens in Worten verbrannt oder durchgeprügelt worden ist. “23

\section{Die Bediensteten}

Die „weiße Industrie“ des Bädertourismus stellte in Westböhmen im 19. und in den ersten Jahrzehnten des 20. Jahrhunderts einen bedeutenden Wirtschaftsfaktor dar. Sowohl für die Kurorte selbst als auch für die umliegenden Dörfer und Städte stellte er eine wichtige Einnahmequelle dar. ${ }^{24}$ Große Teile der Bevölkerung der ländlichen Regionen Westböhmens arbeiteten während der Saison in zahllosen Dienstleistungssparten in den Bädern. ${ }^{25}$ Wie häusliche „Diener" brachten die Angehörigen des Servicepersonals der Bäder zwei Welten zusammen. Sie „dienten“ als „Scharnier oder Mittler zwischen verschiedenen Sphären“ (Krajewski 2008: 21). Dieses „Walten im Dazwischen“ (Krajewski 2008: 21-22) wurde für die böhmischen Bäder bisher kaum bearbeitet.

Mit großer Aufmerksamkeit widmet sich Krackowizer in seinen Aufzeichnungen den Bediensteten der Bäder. Die „Kaffeeheben“, die Brunnenmädchen, der Moorbadediener, die Blumenmädchen, die Oblatenverkäuferinnen, der Hausdiener und das Zimmermädchen finden in seinen Aufzeichnungen immer wieder Erwähnung. Krackowizer beleuchtet deren Arbeitssituation und beschreibt den Umgang mit ihnen, wobei - vor allem was die Kaffeemädchen betrifft, deren Arbeit ihn am meisten zu interessieren scheint - in den Beschreibungen nicht selten ein männlichchauvinistischer Blick aufscheint, etwa wenn er ihr Aussehen beurteilt. So heißt es zum Beispiel: „Ein verblühtes Mädchen bringt lichten Kapuziner und dazu ein Kännlein heißes Wasser“ (Reisetagebuch: 64) oder: „Helena bringt ,Verkehrten“ ohne Schlagobers. Keine schöne Helena!“ (Reisetagebuch: 15)

Sehr aufmerksam beobachtet und notiert er die Arbeit, die die weiblichen Bediensteten in Karlsbad zu leisten hatten. Die Tatsache etwa, dass die Kaffeemädchen körperlich anstrengende Arbeit verrichteten, ohne dass dies besonders geschätzt wurde, die weiten Wege, die viele der im Bad Arbeitenden zurücklegen mussten, um ihrer Arbeit nachgehen zu können, die strenge Aufsicht, denen ihre Arbeit unterlag, sind Themen,

23 Zit. nach Bajohr 2003: 150.

24 „Die Curorte gleichen den Fixsternen, die ihre segenbringenden Strahlen auf Entfernungen aussenden, von denen ein gewöhnliches Auge kaum eine Ahnung hat", heißt es im Bericht der Handels- und Gewerbekammer Eger aus dem Jahre 1859. Bericht 1859: 75-76.

25 Dazu Ruud 1954; Fendl 2011. 
die er anspricht: „Der Mühlbrunnen wird am meisten belagert und die 12 Mädchen bei dieser Quelle haben beide Hände u. 10 Finger stundenlang unermüdlich zu rühren. Bei dem kleinsten Verstoß schreit sie der Aufseher an. Und trotz der unzähligen Becher, die ihnen zum Füllen gereicht werden, niemals eine Verwechslung. Hie und da bricht wohl ein Becher und dann hageln Koseworte in die Tiefe des Brunnens nieder" (Reisetagebuch: 67), berichtet er am 7. Juni 1909, und am 20. Mai heißt es: „Da Manche schon um 1/2 5 Uhr früh trinken, müssen die armen Brunnenmädeln ohne Trinkgelder schon um 4 Uhr bei den Quellen stehen und Becher füllen." (Reisetagebuch: 23)

Auch der große Gegensatz zwischen den vermögenden Kurgästen und der teilweise armen Bevölkerung beschäftigt Krackowizer. Als er z.B. von einem Ausflug zur Glasfabrik Moser in Meierhöfen/Dvory zu Fuß nach Karlsbad zurückgeht, weil er im Omnibus keinen Platz mehr findet, schreibt er: „Auf dem Rückwege [...] lernte ich ärmliche Egerländer Kinder kennen und sah in den Fabrikshöfen die Armut. Ein greller Gegensatz zu dem Luxus des Weltbades." (Reisetagebuch: 26-27)

Die Tatsache, dass die Kaffeemädchen nicht ihre eigenen Namen tragen dürfen, sondern mit „Saisonnamen“ oder gar mit Nummern bezeichnet werden, scheint ihn besonders zu irritieren: „Die Kellnerin No. 5 bedient aufmerksam. Hier haben die Kaffeeheben keinen Saisonnamen auf dem jungfräulichen Busen. Sie legen dem Gaste einfach einen Zettel mit einer Nummer hin. ,Nie sollst du mich befragen!'“ (Reisetagebuch: 9) ${ }^{26}$ Krackowizer äußert sich kritisch zu den zugeteilten Namen, die er als „Marlitt-Namen“ (Reisetagebuch: 31) bezeichnet: „Saisonnamen [im Original unterstrichen] sind: Elsa, Hedwig, Ottilie, Edith, Frieda, Paula, Ida, Emilie. Thekla, Anna oder Maria wäre alltäglich" (Reisetagebuch: 11).

Immer wieder räsoniert er über die anstrengende Arbeit der Kaffeemädchen: „Müde raste ich um $8 \mathrm{Uhr}$ im Freundschaftssaal [im Original unterstrichen]. Kellnerin Elsa geht auf mich zu u. frägt naiv: ,Haben Sie schon Ihr Mädchen?‘, nämlich: zum Bedienen. Bald darauf kommt Elsa und stellt mir ein junges Mädchen vor, die Edith heißt und zum erstenmal heute bedient. Ich bin ihr Versuchskaninchen und mein Trinkgeld ist ihr erstes. [...] Alle tragen schwarze Kleider, zierliche weiße Schürze, eine schwarze Seidenmasche auf dem wohlfrisierten Kopf. Einige Mädchen schleppen Zeitungen zu den Tischen, eine andere wandert mit einer Zigarrenkiste herum. Wie oft im Tage die Mädel die zehn Treppenstufen zur Küche empor laufen, hat kein Statistiker noch berechnet. Kaiserpark [im 


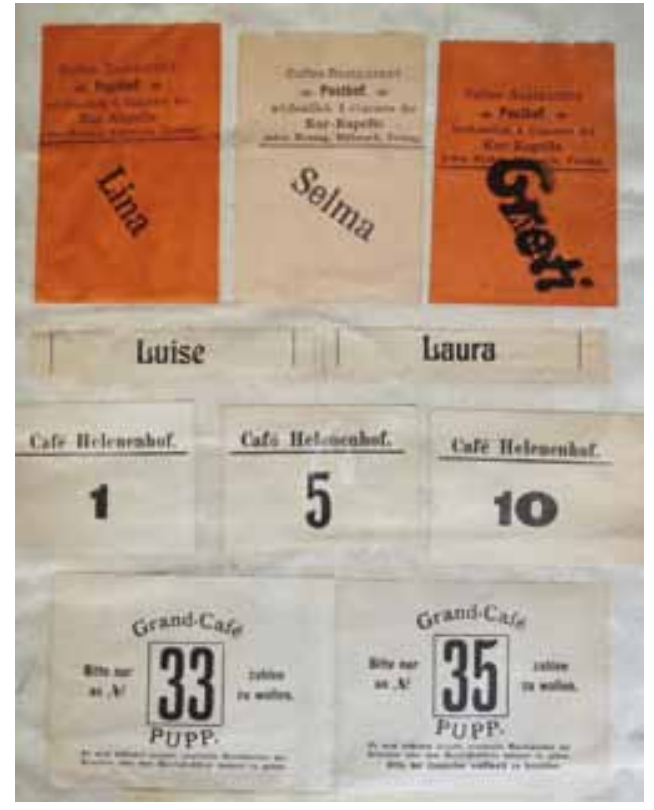

Abb. 9 Servierzettel von Kaffeemädchen aus den Karlsbader Cafés Posthof, Helenenhof und Pupp, Erinnerungsalbum des Ferdinand Krackowizer.

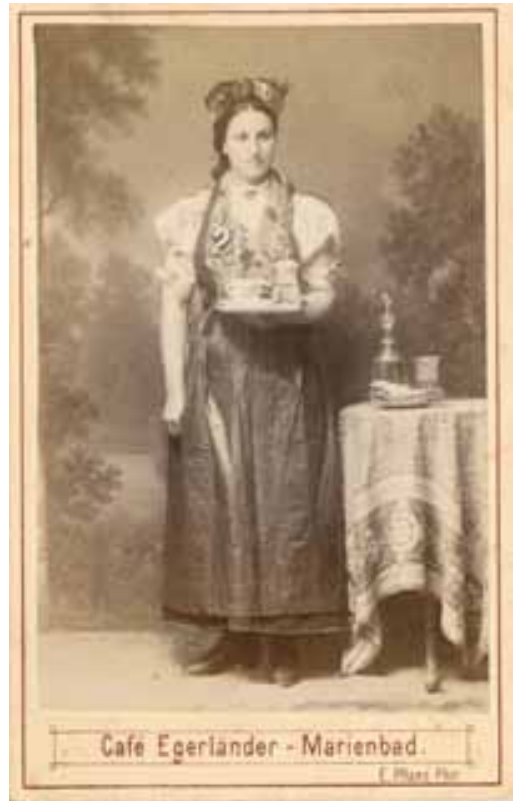

Abb. 10 Kaffeemädchen Nr. 2 des Café Egerländer in Marienbad, Carte de Visite, um 1910.

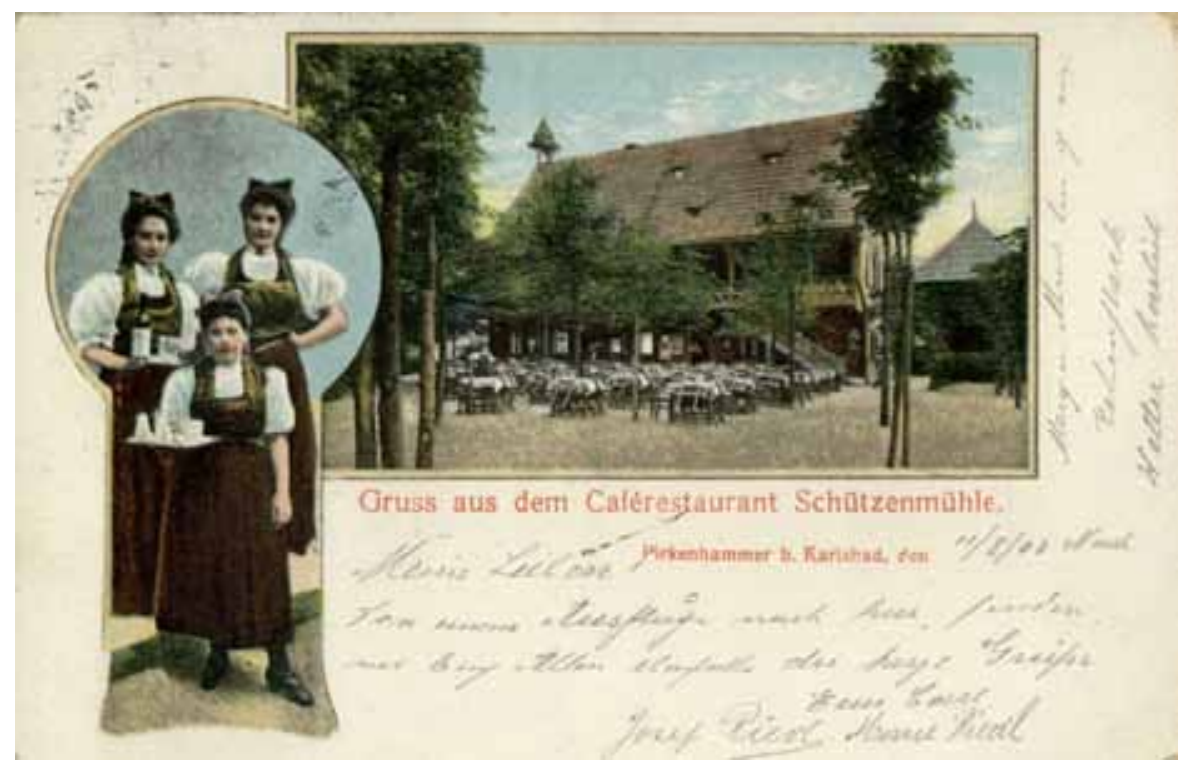

Abb. 11 Bildpostkarte des Caférestaurants Schützenmühle in Pirkenhammer/Březová bei Karlsbad mit Kaffeemädchen in einer Art Egerländer Tracht, gelaufen 1903. 
Original unterstrichen]. Die Mädchen dort sind nummeriert" (Reisetagebuch: 11).

Obwohl Krackowizer - wie andere Kurgäste auch - den Kaffeegenuss einschränken sollte, besuchte er zum Frühstück und zur Kaffeestunde verschiedene Kaffeehäuser und klebte die dazugehörigen Postkarten und Servierzettel, ${ }^{27}$ die mit "Saisonnamen“ und Nummern bedruckt sind und so auf das von ihm beschriebene Benennungssystem verweisen (Abb. 9 und Abb. 10), in sein Album ein.

Die meist aus der Region stammenden Kaffeemädchen, die bei äußert schlechter Bezahlung ${ }^{28}$ und häufig ebenso schlechter Behandlung in den Kaffeegärten der westböhmischen Bäder arbeiteten, trugen teilweise, weil dies Kurgäste anzog, eine Art „Egerländer Tracht“ (Abb. 10 und Abb. 11). Ihre Arbeit wurde so folklorisiert und auch ein Stück weit sentimentalisiert.

Wie anderen Bediensteten auch wurde den Kaffeemädchen ihr angebliches Anbiedern an die Kurgäste und eine bis zur Selbstverleugnung reichende berechnende Unterwürfigkeit vorgeworfen, die nur aus Geldgier geschehe. Auch den Vorwurf der Prostitution mussten sie sich bisweilen gefallen lassen. Die Tatsache, dass die Trinkgelder aber teilweise den einzigen Lohn darstellten und sie deshalb darauf angewiesen waren, wird bei der Kritik ihres Handelns häufig unbeachtet gelassen. Der Journalist und Sozialreformer Max Winter beschrieb 1901 in der Wiener Arbeiterzeitung in zwei Karlsbad-Feuilletons den krassen Gegensatz von Arm und Reich in der Stadt. In einem Absatz über das „System Pupp“ heißt es bei ihm: „Kein Hotelier, kein größerer Hausbesitzer gibt seinen Kellnern und Kellnerinnen, seinen Stubenmädchen und seinen Lohndienern einen Kreuzer Lohn. Sie alle müssen sich mit dem Trinkgeld bezahlt machen, sie alle sind darum kriecherisch, korrumpirt und nicht selten auch - feil." (Winter 1901) Das Trinkgeld als Gegenleistung für freundliches Umsorgen, als „Teil einer kurzfristigen persönlichen Tauschbeziehung“ (Meyer 2011: 261), gewinnt hier eine ganz andere Bedeutung.

Krackowizer zeigt sich in seiner Beurteilung der Situation der Bediensteten der Bäder zwiespältig. Einerseits beobachtet er, wie oben beschrieben, deren hartes Leben, andererseits schreibt er über ein „Vasallenverhältnis“, das ihn mit dem Zimmermädchen und dem Hausdiener des von

27 Wir finden dort Belege des Café Helenenhof (drei Postkarten, von denen eine das Bismarckzimmer zeigt, eine zweite auf den Aufenthalt Bismarcks hinweist und so auch die deutschnationale Haltung des Kurgastes deutlich macht, und ein Servierzettel), des Café Aberg (zwei Postkarten), des Café Freundschaftssaal (eine Postkarte), des Café Pupp (eine Postkarte und ein Servierzettel) und des Café Posthof (Servierzettel).

28 Dazu: Winter 1901. 
ihm gewählten Logierhauses verbinde (Reisetagebuch: 32), und räsoniert über die große Summe an Trinkgeld, die ein Kaffeemädchen während einer Saison verdienen könne: „Laura, der ich Verkehrten ohne Schlagobers zugerufen, bringt mir eine Kanne pechschwarzen Cafés [sic]. Dies kommt ihr selbst thöricht vor und sie holt die dazu gehörige Milch. Solch thörichte Jungfrau verdient sich während der Saison 1000 Gulden Trinkgeld und darüber, geht über Winter heim u. heiratet einen ,Kleinen' scil. [= scilicet - nämlich] Beamten oder Geschäftsmann." (Reisetagebuch: 35)

\section{Die Kuranwendungen und die Folgen}

Auffallend häufig berichtet Krackowizer in seinem Tagebuch über seinen körperlichen Zustand. Er beschreibt die Last des Trinkens von drei Bechern Wasser am frühen Morgen, beschwert sich über die Hitze und das Gewicht des Moorumschlags und beklagt sein durch die Diät bedingtes Hungergefühl. Auch ein ansonsten wohl weitgehend tabuisiertes Thema wird immer wieder angeschnitten, das der Verdauung. Dabei gefällt sich Krackowizer darin, immer neue Umschreibungen für diesen Vorgang zu finden. So heißt es etwa am 19. Mai 1909: „Auflösend auf den Leib aber wirkt die Felsenquelle, welche viele Gäste sich holen lassen, um die Eröffnungsvorstellung am Morgen glänzend auszustatten u. durchzuführen“ (Reisetagebuch: 19) und 10 Tage später: „Ebenso glatt verläuft die Besteigung des Thrones der Pythia. Auch sehr wichtig. Wird auch der Herr ,Geheimbde Rat' von Goethe getan haben." (Reisetagebuch: 50) Und selbst was den Toilettengang betrifft, beschäftigt Krackowizer das Geld: „Die Pförtnerin der Pfortenkabinen nimmt mehr Trinkgeld ein, als mancher fleißige Journalist im Tage seiner Feder verdankt" (Reisetagebuch: 31-32).

Das Sprechen über die Ausscheidungen des menschlichen Körpers wurde, so Annemarie Hürlimann, seit der Neuzeit strengen Regeln unterworfen: „So hervorgerufene Scham- und Peinlichkeitsgefühle verlagerten das fäkalische Reden und Tun allmählich von der Öffentlichkeit in die private Sphäre, vom Alltag in die Medizin und später in die Psychoanalyse. "Nur mehr in derben Witzen und in Karikaturen hätten Trieb- und Affektkontrollen „tabubrechende Befreiung“" gefunden (Hürlimann 1996). Im Umfeld von Kurorten - wie auch Belege aus anderen Bädern zeigen - wird das Thema jedoch häufiger als anderswo angesprochen. Für Karlsbad gibt es eine nicht geringe Anzahl von Bildpostkarten, die das Thema der Verdauung aufnehmen und vor allem die kräftige Wirkung der Karlsbader Wässer behandeln (Abb. 12). 


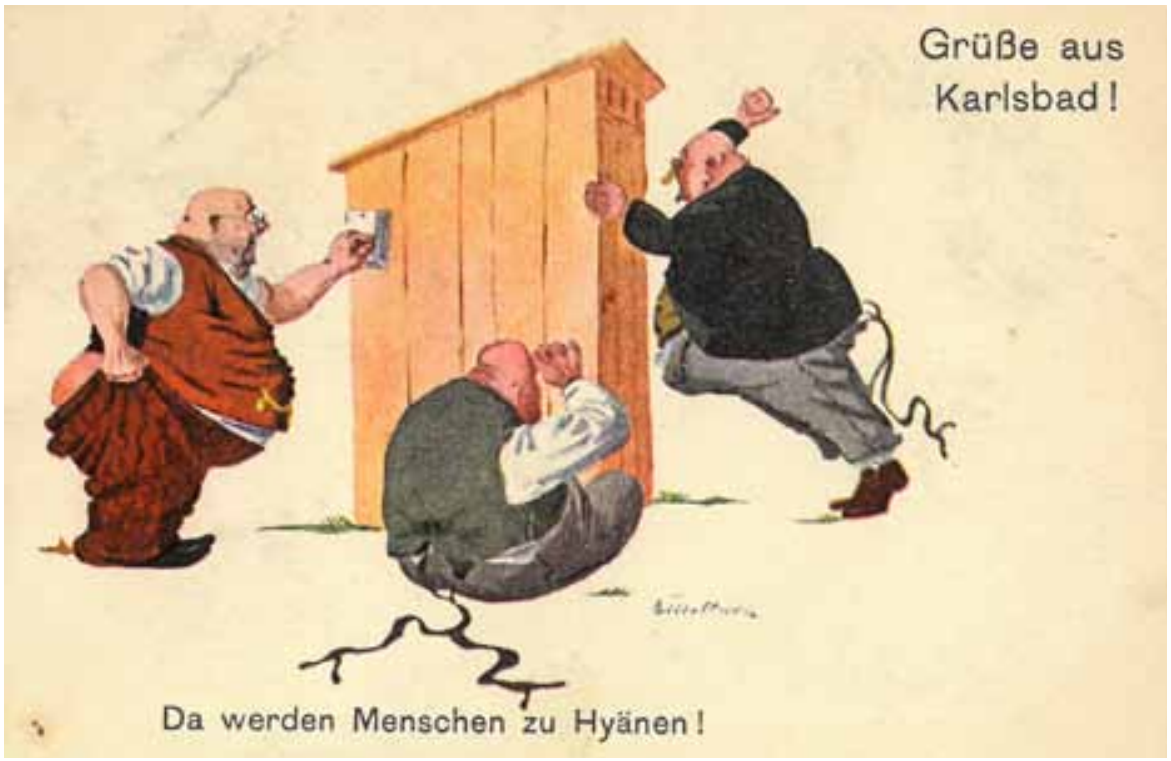

Abb. 12 „Humorkarte“ aus Karlsbad, Verlag Brüder Kohn, Wien, ungelaufen, um 1910.
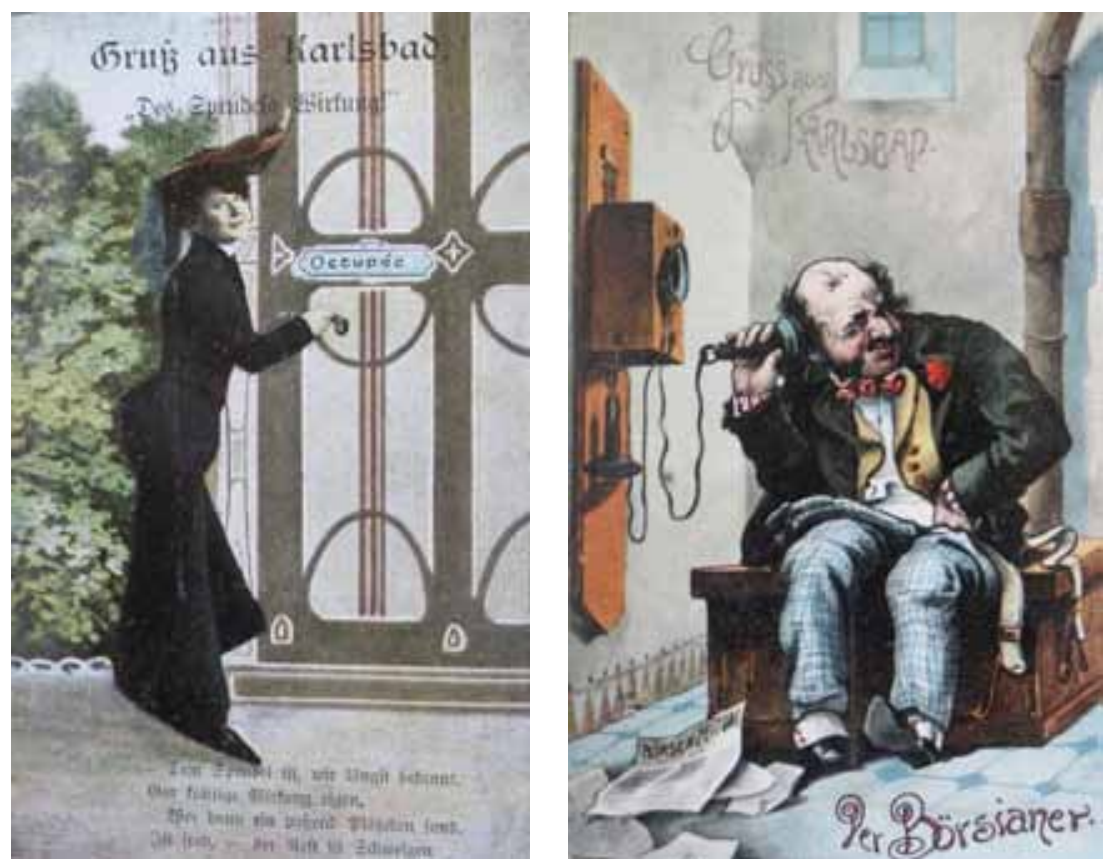

Abb. 13 und Abb. 14 Bildpostkartenpaar zum Thema Verdauung im Erinnerungsalbum Krackowizers. 
Die unter diesen zu findenden judenfeindlichen Postkarten sind - so Klein - die ,infamsten der explizit antisemitischen Karten aus den böhmischen Bädern" (Klein 2006: 159).

Wie wir einem Postkartenpaar im Album Krackowizers (Abb. 13 und Abb. 14) entnehmen können, wird bei der Darstellung des Themas Verdauung böse differenziert: auf der einen Postkarte ist eine elegante Dame zu sehen, die verschämt bis kokett die Türklinke einer als besetzt markierten Toilettentür drückt, dieser Karte gegenübergestellt ist eine Karte mit aggressiv antisemitischem Charakter. Ein als „Börsianer" bezeichneter, seiner Physiognomie nach als Jude gekennzeichneter Mann wird auf der Toilette sitzend bei seinen im doppelten Sinne schmutzigen Geschäften vorgeführt (Dipper 1999: 199).

\section{Positionierungen}

„Bei Pupp etwas fade. Langweiliges Publikum mit unschönen deutschen Weibervölkern. Bei der Verande [sic] liegen rote Plaid zum Einhüllen bereit. Am Eingang lauern die schönfrisierten Mädchen. No. 51 bringt guten ,Verkehrten'. Viel heiterer ists im Freundschaftssaal. [...] Keine Zeitung zu kriegen! Mich u Pupp, kriegst auch nicht oft zu sehen" (Reisetagebuch: 42) - Mit Bemerkungen wie dieser versucht Krackowizer sich in seinem Reisetagebuch immer wieder von den vom ihm als "Gecken" (Reisetagebuch: 20) bezeichneten neureichen Kurgästen abzusetzen. Er, der lieber die Ruhe der Waldwege suchte, distanziert sich von den „Genußmenschen“ und ihren Vorlieben: „Unten aber im Tale der Tepl rollen unaufhörlich Omnibusse, Einspänner, flotte Wiener Fiaker, elegante Herrschaftskutschen und lärmende Autos - mit Genußmenschen, oben im Grün die Poesie! Die Amseln in ihrer Liebessehnsucht sind unerschöpflich im Flötensolo“ (Reisetagebuch: 57), schreibt er am Pfingstmontag 1909, und bereits einige Tage vorher hatte er notiert: „Nach dem Genuße der einzig schönen Waldwege ist der Welttrubel der Gecken aller Länder und der aufgedonnerten Modeweiber, die vom eleganten Café Pupp und der Alten Wiese bis zur Kolonnade bummeln, ein starker Gegensatz. Die Warenpreise in den dortigen Schaubuden sind unnatürlich in die Höhe geschraubt." (Reisetagebuch: 20) Für Naturliebhaber, die „Erholung von dem Treiben der Städte“ suchten, wurden Kurorte, die so viele vermögende Kurgäste anzogen, „ebenfalls [...]“, so Prein, „zum Sinnbild mondäner Urbanität“ (Prein 2005: 161). Das belegen auch die Notizen Krackowizers. Er beschreibt diesen Zwiespalt jedoch nicht nur, er setzt seine bildungsbürgerlichen Interessen über die vermeintlich vor allem auf Konsum und Prahlerei ausgerichteten Interessen anderer Kurgäste. Während er über den Aufenthalt Stifters in Karls- 
bad im dortigen Museum Forschungen betreibe, immer wieder zu Stifters Wohnhaus „Zwei Prinzen“ spaziere und häufig Konzerte besuche, fänden andere - so vermittelt er - an seichten Vergnügungen Spaß. Mit dieser Selbstdarstellung reiht er sich ein in die Reisenden, die - Konrad Köstlin hat dies beschrieben - etwa auf den Spuren Goethes nach und durch Böhmen reisten (Köstlin 1992; Köstlin 1994). Den Wegen des großen Deutschen nachzugehen, den „von Goethe gepriesenen und von unzähligen Epigonen nachempfundenen Blick“" zu erleben (John 1907: 12), schien in der 2. Hälfte des 19. Jahrhunderts einer religiösen Erfahrung gleichzukommen: nationale Weihegänge, nationale Wallfahrten auf Wegen, die markiert waren und so zu Lehrpfaden und „Lernpfaden des nationalen Empfindens“ (Köstlin 1992: 23) wurden. Für den Oberösterreicher Krackowizer war es eher Stifter, dem er „nachfolgte“. Immer wieder erwähnt er dessen Aufenthalt in Karlsbad und notiert enttäuscht: „Schade, daß er [Stifter] über Karlsbad keinen Aufsatz schrieb" (Reisetagebuch: 33).

\section{Die Architektur der Bäder}

An der Stadt interessierten Krackowizer erst in zweiter Linie die Geschäfte und Restaurants, mehr noch scheint er an der Architektur Gefallen gefunden zu haben. So berichtet er vom Villenviertel Westend, beschreibt einen Aufenthalt in der russisch-orthodoxen Kirche, besucht die Friedhöfe der Stadt und lässt sich durch das Kaiserbad führen. ${ }^{29}$

Je mehr Badegäste aus dem wohlhabenden Bürgertum Karlsbad besuchten, umso mehr Neubauten wurden erforderlich. Neben der zum medizinischen Kurbetrieb gehörenden Architektur der Kolonnaden, der Brunnentempel und Badehäuser mussten auch „Konversationsräume“, Theater, Kirchen errichtet werden. Über die Bildpostkarte als „erstes visuelles Massenmedium“ wurden die Blicke auf diese kurgerecht konstruierten Stadt-Landschaften weltweit verschickt (Telesko 2014: 32).

Das anspruchsvolle Publikum verlangte nach repräsentativen Hotelbauten. Palastartige Hotelkomplexe wie das Grand-Hotel Pupp ${ }^{30}$ oder das Hotel Imperial ${ }^{31}$ boten den vermögenden Gästen die Möglichkeit, während des Kuraufenthaltes den mondänen Lebensstil der Aristokratie

$29 \mathrm{Ob}$ es Zufall ist, dass Krackowizer die architektonisch interessante Karlsbader Synagoge, 1877/78 errichtet von dem Stuttgarter Architekten Adolf Wolff (dazu Schwarz 1988), nicht erwähnt, oder ob das sein Desinteresse an der jüdischen Kultur oder gar seine Verachtung dieser Kultur belegt, ist nicht zu sagen.

$30 \mathrm{Zu}$ Geschichte und Mythos dieses Hotels Fendl 2002.

31 Nach Plänen von Ernest Hébrard wurde das Hotel in den Jahren 1910 bis 1912 errichtet. Es stellt den letzten großen Hotelneubau der K.u.K. Monarchie dar. 


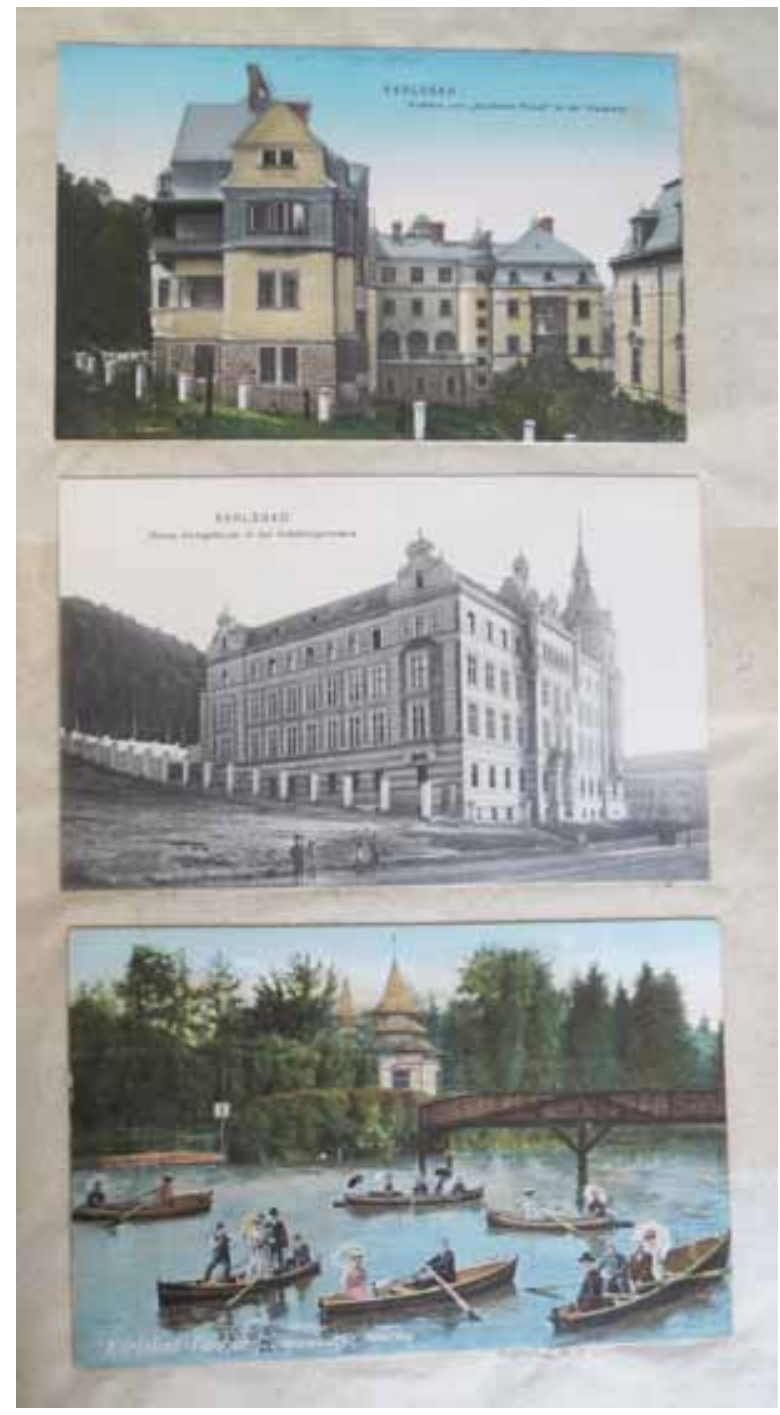

Abb. 15 Seite aus dem Erinnerungsalbum Krackowizers mit Bildpostkarten des Kurhauses „Zum Goldenen Kreuz“, des neuen Amtsgebäudes in des Habsburgerstraße und des Knoll'schen Teepavillons in Fischern.

nachzuahmen. Einen Höhepunkt den Bau palastartiger Hotelkomplexe betreffend stellte der Bauboom der Jahre 1900 bis 1914 dar (Schmitt 1982: 11; Wenzel 1991). Im Grandhotel trat - so Ernst Wilhelm Heine - ein Grundzug des Zeitalters zutage: "Jeder will mehr darstellen, als er ist, und mehr besitzen, als er sich leisten kann. ${ }^{32}$ Dieses Mehr-Scheinen-als-

32 Zit. nach: Bien - Giersch 1989: 34. 
Sein, das hier für die Architektur ausgemacht ist, kann als ein Prinzip beschrieben werden, das auch viele andere Bereiche des Lebens im Kurort bestimmte.

Die Architekten, die das Karlsbad der Jahrhundertwende am deutlichsten prägten, waren die Wiener Ferdinand Fellner und Hermann Helmer, die von 1873 bis 1919 in einem Atelier zusammenarbeiteten. Ihre Zentralbauten waren in der gesamten Monarchie vertreten. In Karlsbad errichteten sie u. a. den Neubau des Theaters (1886), das als Kaiserbad bezeichnete Bad I (1895 eingeweiht), Teile des Grand-Hotels Pupp (1896-1913), die Sprudelkolonnade (1878-1879) und das Schützenhaus (Zídková 1996). Wie Postkarten in seinem Erinnerungsalbum und Notizen im Reisetagebuch belegen, beeindruckten Krackowizer neben diesen Bauten so unterschiedliche Bauwerke wie die 1900 im neogotischen Stil errichtete Ecce-Homo-Kapelle (Gnirs 1996: 56), der 1945 zerstörte Japanische Teepavillon des Porzellanfabrikanten Carl Knoll (Zerlik 1983) (Abb. 15), aber auch das neue Amtsgebäude in der Habsburgerstraße (Abb. 15). Dass er an neuer Architektur interessiert war, zeigt etwa auch die Postkarte des Beamten-Kurhauses „Zum goldenen Kreuz" in der Waldzeile (Abb. 15), das in den Jahren 1906 bis 1907 von dem Otto Wagner-Schüler Wunibald Deininger errichtet worden war (Vybíral 2007: 209).

Krackowizers technisches Interesse belegt die Fahrt mit der im Mai 1907 eröffneten ersten Standseilbahn in Karlsbad (Abb. 16), die unterirdisch vom Theaterplatz zum Café Helenenhof führte, das unterhalb des 1912 eröffneten Hotels Imperial lag. ${ }^{33}$

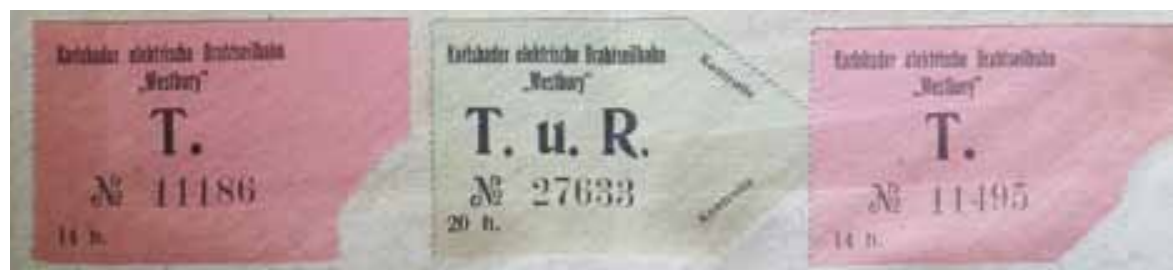

Abb. 16 Karten für die Fahrt mit der Karlsbader Drahtseilbahn im Erinnerungsalbum Krackowizers.

33 Die Seilbahn führt über eine $127 \mathrm{~m}$ lange Strecke auf eine Höhe von $437 \mathrm{~m}$. Dazu: Wikipedia Artikel Standseilbahnen in Karlsbad; Hubínek 2013 - 1980 wurde die Bahn zunächst stillgelegt, nach Renovierungsarbeiten zwischen 1983 und 1987 wurde sie im November 1987 wiedereröffnet. 
Die Entdeckung des Kurortes und seiner Umgebung

Anders als die adeligen Kurgäste früherer Zeiten, bezog das ins Bad reisende Bürgertum auch die Umgebung des Kurortes in seinen Bewegungsradius mit ein. Die Reisehandbücher des 19. und frühen 20. Jahrhunderts liefern mannigfaltige Vorschläge für Spaziergänge und Ausflüge in die Umgebung des Kurortes.

Zum Ausflugsprogramm Krackowizers gehörten außerhalb Karlsbads, neben dem Besuch der Porzellanfabrik Carl Knoll in Fischern/Rybáře und der 1857 gegründeten Glasfabrik Moser in Meierhöfen/Dvory, auch die Felsformation der Hans-Heiling-Felsen / Svatošské skály und die Stadt Elbogen/Loket.

Das "Weiße Ross" in Elbogen (Abb. 17) etwa war für ein bildungsbürgerliches Publikum unter anderem deshalb zur Destination geworden, weil Goethe hier verschiedentlich Gast war ${ }^{34}$ und zum Beispiel 1823 mit Ulrike von Levetzow, ihrer Mutter und ihren beiden Schwestern seinen 74. Geburtstag feierte. Daneben bot die mittelalterliche Stadt mit ihrer Burg, dem seiner Bestände wegen bedeutenden Stadtmuseum, der Porzellanfabrik und der 1836 eingeweihten Kettenbrücke gleich mehrere touristische Orte, die bereits im letzten Drittel des 19. Jahrhunderts erprobt waren..$^{35}$ Die „imposante Kettenbrücke [...] d die“ - so Leopold Fleckles 1838 - „rücksichtlich ihrer Höhe und der Masse des Mauerwerkes in dem österreichischen Kaiserstaate nichts Aehnliches hat" (Fleckles 1838: 68) ${ }^{36}$ wird von Krackowizer mit heimischem Maßstab bewertet. "Joche doppelt groß wie b. d. [bei der] Tauernbahn" (Reisetagebuch: 60) - heißt es im Reisetagebuch lapidar. Größeres Interesse erregte bei ihm das im Stadtmuseum verwahrte Stück eines Eisenmeteoriten aus dem 14./15. Jahrhundert, der als „verwunschener Burggraf“ in die populären Erzählstoffe Westböhmens Eingang gefunden hatte: „Der berühmte Meteor [im Original unterstrichen], noch ein großes Stück. Der ganze Meteor nur im Nachguß, weil zur Verteilung an Museen von Kaiser Franz bestimmt" (Reisetagebuch: 60).

Wie zwei ins Erinnerungsalbum Krackowizers eingeklebte Postkarten (Abb. 18) mit kolorierten Fotografien des Karlsbader Heimatkundlers

34 Eine am Haus angebrachte Steintafel notierte Besuche 1807, 1808, 1810, 1811, 1818, 1819 und 1823. Dazu: https://www.boehmisches-erzgebirge.cz/pictures/ Elbogen/ (26.03.2020).

35 Vgl dazu etwa Schönpflug 1875: 63-64.

36 Fleckles widmet diesem Bauwerk in seinem Führer sechs Seiten, dazu Fleckles 1838: 68-73. 


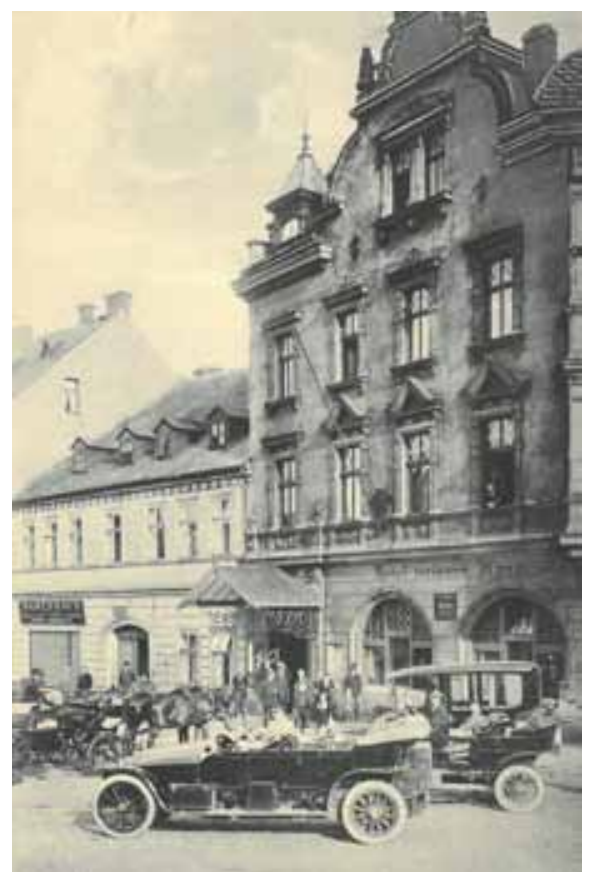

Abb. 17 Werbe-Fotopostkarte des Hotelrestaurants „Weisses Ross“ in Elbogen, um 1910.

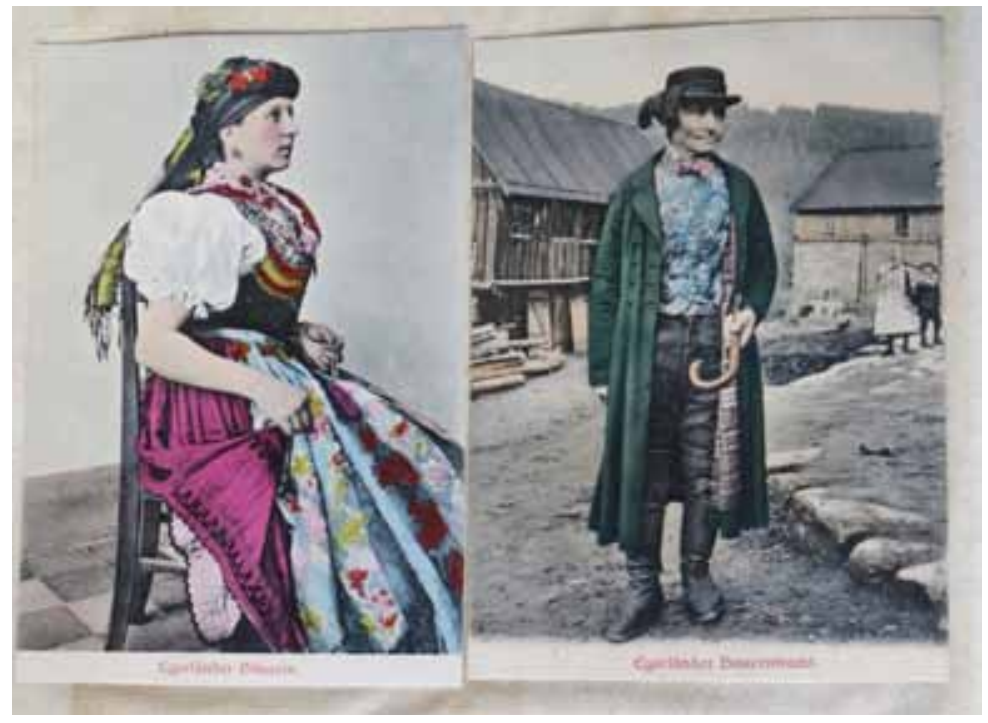

Abb. 18 Fotopostkarten „Egerländer Bäuerin“ und „Egerländer Bauerntracht“ mit kolorierten Fotografien von Josef Hofmann, um 1905. 
Josef Hofmann ${ }^{37}$ zeigen, scheint er auch Interesse am umgebenden Egerland gehabt $\mathrm{zu}$ haben. Wie seine kurzen Bemerkungen dazu zeigen, stellte das Land für ihn einen Gegenentwurf zur Stadt dar. Da ist von „frische[n] Egerländerinnen“ die Rede, die Mineralwasser zum Versand abfüllten (Reisetagebuch: 12), da werden die Kaffeemädchen des Posthofs als „brav[e]“ Egerländerinnen beschrieben (Reisetagebuch: 49), da schwärmt er bei seinem Gang über den Karlsbader Friedhof von der schönen Aussicht ins „fruchtbare Egerland“ (Reisetagebuch: 21).

Wie Krackowizer das Land als Gegenentwurf diente, diente ihm die Tracht als „Symbol der Gegenläufigkeit“ (Köstlin 1988: 305), als Fluchtpunkt auch. Als Zeichen eines einfachen und natürlichen Gemeinschaftslebens, das frei von allen Konflikten der Industriegesellschaft war, wurden die Postkarten - so ist anzunehmen - in sein Erinnerungsalbum aufgenommen.

\section{Die Kosten der Reise}

Die hohen Kosten, die der Aufenthalt in Karlsbad verursacht, werden im Reisetagebuch immer wieder angesprochen. Bereits mit zwei seinen Aufzeichnungen aus Karlsbad als Motti vorangestellten Zitaten wird darauf angespielt. Da heißt es zum einen mit Shakespeare: „Tu' Geld in deinen Beutel $[\ldots]$ “, zum anderen wird ein auf „Karlsbader Tassen“ zu findender Spruch zitiert: „Frau, ärgere nicht deinen Mann, / die Kur kostet Geld.“

Das erste Zitat wird auch im Karlsbad-Führer von Josef Ruff aufgeführt, wenn der Autor Ratschläge zur Vorbereitung der Reise gibt. Ruff stellt es jedoch in den größeren Zusammenhang von Badereisen im Allgemeinen: „Zunächst erinnern wir daran, was Jago dem Cassio empfohlen hat: ,Tue Geld in Deinen Beutel!' Dieses Zitat gilt für jeden, der in eine Kur geht und darf nicht die Deutung erfahren, als ob Karlsbad teurer wäre als irgend ein anderer Kurort; man lebt hier so billig oder so teuer wie man will, aber Sparsamkeit ist in einem Kurorte überhaupt schlecht angebracht." (Ruff 1904: 49)

Gerade in Karlsbad angekommen, notiert Krackowizer am 15. Mai 1909: „Und diese prachtvollen Auslagen. Und jetzt schon ein großer Fremdenverkehr. Aber ein teures Pflaster!" (Reisetagebuch: 2) und am 16. Mai 1909: „Aber teuer kommt der Aufenthalt hier“ (Reisetagebuch: 4). Anlässlich eines Waldspaziergangs am 31. Mai 1909 heißt es: „Der Kukuk [sic] ruft, das Moos nimmt ab" (Reisetagebuch: 57). Dabei ist es vor allem

37 Bei Josef Hofmanns Fotografien handelte es sich um gestellte Fotografien, die einen Zustand der Tracht zeigten, die zum Aufnahmezeitpunkt nicht mehr aktuell war. Zu den „Feldforschungen“ Hofmanns Fendl 1995. 


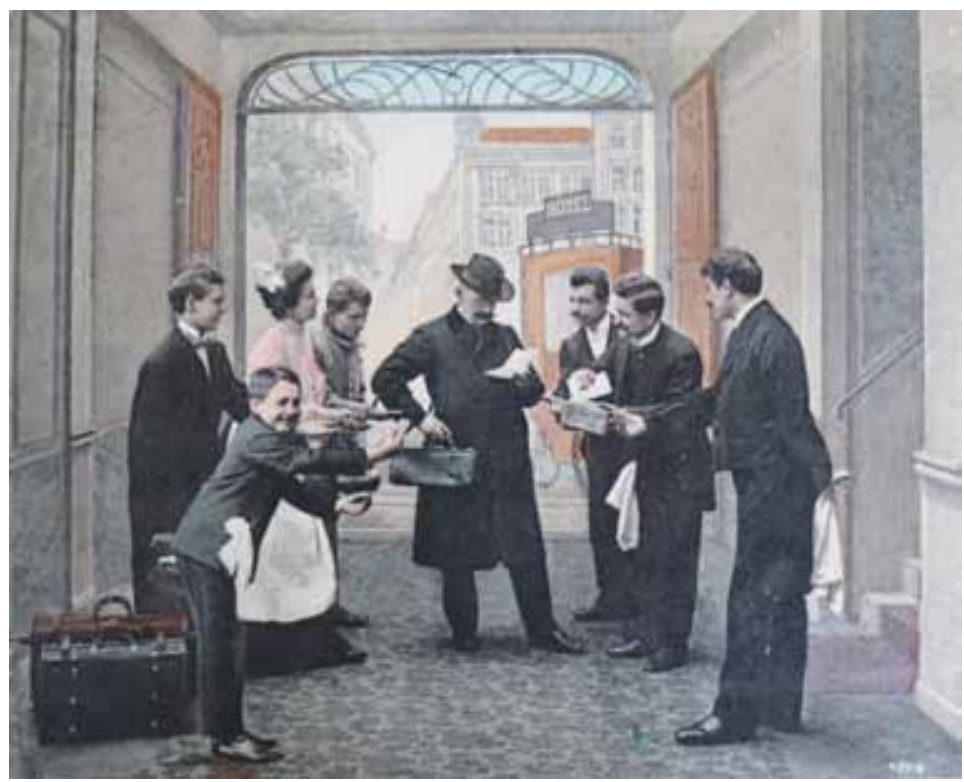

Abb. 19 Bildpostkarte mit Darstellung eines die Rechnung kontrollierenden abreisefertigen Gastes, umringt von Trinkgeld heischenden Bediensteten, um 1905.

das an verschiedene Bedienstete zu zahlende Trinkgeld, das immer wieder betont wird: „Zu jeder Mahlzeit ist bekömmlich für den Ober $20 \mathrm{~h}$, für den Zuträger der Speisen $20 \mathrm{~h}$ und $10 \mathrm{~h}$ für den Piccolo. Daher kosten 2 Mahle im Tage 1 K Douceur!“ (Reisetagebuch: 4), oder an anderer Stelle: „Die Trinkgelder verteuern die Sachen unglaublich" (Reisetagebuch: 25).

Eine in sein Erinnerungsalbum geklebte Bildpostkarte ${ }^{38}$ (Abb. 19) zeigt einen Gast, der bei der Abreise von mehreren Bediensteten belagert und um Trinkgeld gebeten wird. Das Motiv, das teilweise die Bezeichnung „Abreise“ oder „Départ" trug, war im frühen 20. Jahrhundert in mehreren Varianten sehr beliebt, nicht nur auf den in großer Zahl in den Bädern verkauften Karten. ${ }^{39}$ Damit wird deutlich, dass das Beklagen hoher Kosten nicht auf Kuraufenthalte beschränkt war. Zu bedenken ist dabei auch,

38 Weil Krackowizer die Postkarten in sein Erinnerungsalbum fest eingeklebt hat, sind bei dieser, wie bei den meisten anderen Karten aus dem Album, keine genauen Angaben zu Verlagen und Entstehungszeit zu machen.

39 Auch eine aus der Frankfurter Zeitung übernommene Notiz im „Karlsbader Kurgast" mit dem Titel „Wörterbuch mit Reisende" nimmt auf diese Szene Bezug, wenn das „Départ“ der Gäste „plötzliches Erscheinen aller Bediensteten“ übersetzt wird. Dazu: Humoristisches. Aus dem Wörterbuch für Reisende, in: Karlsbader Kurgast, 1912, Nr. 18, Beilage, S. 1. 
dass das Kokettieren damit, was man für die Reise ausgegeben hat, ja ungesagt auch den Verweis darauf einschließt, wie viel man sich hat leisten können bzw. was einem die jeweilige Reise wert war.

\section{Krackowizers Netzwerke}

Beschreibt man mit Hermann Sommer das Dasein als Kurgast als eine „aus dem Alltag herausgelöste[ ] Lebensweise“ (Sommer 1999: 8), so stimmt das in dem hier behandelten Fall nur bedingt. Wie das Verhalten Krackowizers zeigt, wurden auch während des Kuraufenthaltes vielfältige Kontakte zum heimatlichen Umfeld gehalten.

Als Ferdinand Krackowizer in Karlsbad ankam, war er der 21. aus Linz stammende Gast, der hier kurte. Bereits im Schnellzug von Linz nach Budweis/České Budějovice traf Krackowizer den Linzer Kreisgerichtspräsidenten i.R. Franz Höss ${ }^{40}$ der sich ebenfalls auf dem Weg nach Karlsbad befand. Am ersten Kurtag begegnete er dem Ackerbauschuldirektor Johann Mainzer ${ }^{41}$ aus Marchtrenk und der oben bereits erwähnten Johanna Dimmel aus Linz. Der Linzer Oberbaurat Leopold Petri ${ }^{42}$ gehörte ebenso zu seinem Umgang in Karlsbad wie verschiedene Mitglieder der Familie Straberger, Juristen und Privatiers aus Wels und Urfahr. ${ }^{43}$ Die Oberösterreich-Runde ergänzten außerdem die Ordensschwester Grete Maschl, ${ }^{44}$ Dr. Karl Platzer, ${ }^{45}$ das Ehepaar Scheinig ${ }^{46}$ der Apotheker Otto Hauk, ${ }^{47}$

40 Karlsbader Kurliste 1909, Nr. 91, 17. Mai 1909, unter Nr. 6782: „Herr k.k. Hofrat und Kreisgerichtspräsident i. P. Franz Höss, Linz, Goldenes Kreuz, Waldzeile".

41 Karlsbader Kurliste 1909, Nr. 51, 6. Mai 1909, unter Nr. 3633: „Herr Ackerbauschuldirektor i. P. Johann Mainzer, Marchtrenk, Moltke, Gartenzeile“.

42 Karlsbader Kurliste 1909, Nr. 92, 18. Mai 1909, unter Nr. 6888: „Herr k.k. Oberbaurat Leopold Petri, Linz, Rosenstock, Kreuzstrasse“.

43 Karlsbader Kurliste 1909, Nr. 91, 17. Mai 1909, unter Nr. 6818, 6820, 6821 und 6822: „Herr Priv. Viktor Straberger mit Frau Julie, Wels, Zwei Prinzen, Kirchenplatz“, „Herr k.k. Gerichtsadjunkt i. P. Theodor Straberger, Wels, Zwei Prinzen, Kirchenplatz“, „Herr k.k. Landgerichtsrat i. P. Alfred Straberger, Urfahr, dto.“ Und „Frl. Privatiere Hermine Straberger, Wels, dto.“.

44 Karlsbader Kurliste 1909, Nr. 92, 18. Mai 1909, Nr. 6890: „Ordensschwester Grete Maschl, Linz, Fürstenhof, Parkstrasse“.

45 Karlsbader Kurliste 1909, Nr. 91, 17. Mai 1909, Nr. 6750: „Herr Med. Dr. Karl Platzer, Steyr, Hotel Nürnb. Hof, Kirchenstrasse“.

46 Karlsbader Kurliste 1909, Nr. 118, 23. Mai 1909, Nr. 8907: „Herr Direktor Franz Scheinig mit Frau Anna, Linz, Diamant, Egerstrasse“.

47 Karlsbader Kurliste 1909, Nr. 121, 24. Mai 1909, Nr. 9088: „Herr Apotheker Otto Hauk, Linz, Stadt Odessa, Egerstrasse“. 
das Ehepaar Schott, ${ }^{48}$ Baronin Hornstein ${ }^{49}$ Hermine Sonnleither ${ }^{50}$ und Baron Grammont. ${ }^{51}$

Wie man dem Reisetagebuch entnehmen kann, suchte Krackowizer anscheinend regelmäßig in der Kurliste nach eingetroffenen Oberösterreichern. Im Reisetagebuch lesen wir zum Beispiel unter dem 23. Mai 1909: „Neuangekommen, der Direktor der Linzer Tramway Scheinig sammt Frau." (Reisetagebuch: 31)

Die Rolle der Kurlisten als Kommunikationsmittel ist bisher zu wenig beachtet worden, obwohl ihr eifriges Studium in Reiseberichten und Feuilletons immer wieder beschrieben wird. 1843 heißt es bei Julius Ries: „Erpichter noch als die Engländer nach ihren meilenlangen Tagesblättern, sind die meisten Kurgäste nach der Kurliste; sie zählen fast die Minuten bis sie erscheint [...]." (Ries 1843: 52) Und auch Marie von Ebner-Eschenbach kommentierte in ihren Episteln aus Franzensbad: „Die Kurliste wird eifrig und täglich studirt, wir kennen den Namen, die Heimath, den Stand eines jeden Badegastes, seine Wohnung, die Zahl seiner mitgebrachten Diener, wir schließen auf seinen Reichthum oder seine Großmuth nach der Summe, die er zu den gemeinnützigen Sammlungen beigetragen, wir erforschen, weshalb er gekommen, wie lange er bleibt [...] (Ebner-Eschenbach 1858: 95-96).

Auch vor Ort lebende Oberösterreicher wurden in das Netz eingefügt, so die aus Linz stammende „Direktrice“ des Kaiserbades, die Krackowizer eine exklusive Führung durch den 1895 eröffneten Komplex ermöglichte (Reisetagebuch: 46), oder der Besitzer des Café Freundschaftssaal, Martin Schmalwieser, über den es heißt: „Der Herr des Freundschaftssaales [im Original unterstrichen], der edle Mostindianer Schmalwieser, verehrt mir einen kleinen Führer, den er, subventioniert" habe" (Reisetagebuch: 31). Die Tatsache, dass der Kaffeehausbesitzer mit dem Regional-Spottnamen „Mostindianer" belegt wird, einer Bezeichnung, die Krackowizer neben Mostschädel auch als Eigenbezeichnung verwendet, macht ihn zu einem, der dazu gehört.

Neben dem als Teil seines Kuralltags zu verstehenden Netzwerken in Karlsbad bestimmte das Kontakthalten mit der Heimat bzw. den Daheim-

48 Karlsbader Kurliste 1909, Nr. 91, 17. Mai 1909, Nr. 6773: „Herr Verwalter Theodor Schott mit Frau Käthe, Linz, Diamant, Egerstrasse“.

49 Karlsbader Kurliste 1909, Nr. 140, 29. Mai 1909, Nr. 10636: „Frau Baronin Hornstein-Bussmannshausen mit Tocht. Antoinette, Linz, Insel Malta, Kaiserstrasse".

50 Karlsbader Kurliste Nr. 91 vom 17. Mai 1909, S. 2 unter Nr. 6819 „Frau Kaufmann Hermine Sonnleitner, Wels, Zwei Prinzen, Kirchenplatz".

51 Karlsbader Kurliste 1909 Nr. 146 vom 1. Juni 1909, S. 2 unter Nr. 11077 „Herr Priv. Rudolf Baron Grammont, Linz, Weilburg, Gartenzeile“. 
gebliebenen den Aufenthalt Krackowizers in Karlsbad. Wie er in seinem Reisetagebuch aufführt, hat er während der dreiwöchigen Kur 70 Karten geschrieben, obwohl er das „Schmieren von Ansichtskarten“ als „Unsitte“ bezeichnet, die ihm bei seiner „ausgedehnten Bekanntschaft" viele Stunden raube (Reisetagebuch: 30). Unter den Angeschriebenen befanden sich neben Familienangehörigen Honoratioren der Stadt Linz und Kollegen aus anderen österreichischen Städten. ${ }^{52}$

Während das Kartenschreiben im Tagebuch als lästiger, zeitraubender

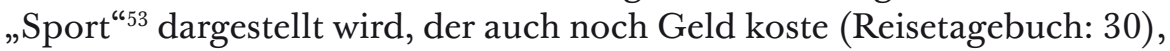
werden der Erhalt von Briefen und Postkarten aus der Heimat stets als große Freude, ${ }^{54}$ als „Dessert “ des Tages auch beschrieben. ${ }^{55}$

Als weiteres Band in die Heimat diente Krackowizer die Linzer TagesPost, die er sich nach Karlsbad schicken ließ. In seinem Reisetagebuch notierte er auch, wo man in Karlsbad Zeitungen aus Oberösterreich lesen konnte: „Der Kaffetier Schmalwieser, ein Landler aus Offenhausen, hält die Tagespost, sowie den Welser Anzeiger" (Reisetagebuch: 16).

\section{Zum Schluss}

Ohne die Analyse weiterer, ähnlicher Quellen wäre es zu gewagt, die Badereise des der bildungsbürgerlichen Elite angehörenden Archivrates Ferdinand Krackowizer als paradigmatisch für eine Form des sich seit dem späten 19. Jahrhundert zum Massenphänomen entwickelnden Reisens einer bürgerlichen Oberschicht zu bezeichnen. Was festgehalten werden kann, ist, dass Krackowizers Reise bzw. die Darstellung dieser Reise geprägt war vom steten Bezug auf bürgerliche Werte und Normen. Die „Tugenden“ Fleiß, Sparsamkeit und Disziplin etwa bestimmten seinen Kuraufenthalt und das Schreiben darüber. Neben gesundheitlichen Beweggründen für die Reise ins Bad lassen sich auch bei Krackowizer gesellschaftliche, Status stabilisierende Gründe ausmachen.

52 So erhielten u.a. der Wiener Botaniker Heinrich von Handel-Mazzetti, der damalige niederösterreichische Minister für Kultus und Unterricht Dr. Gustav Marchet, der Wiener Kunstprofessor Vinzenz Leicht-Lyckdorff, der Direktor des Salzburger Priesterseminars Dr. Antonius Keil und der Leiter der Linzer Studienbibliothek Dr. Konrad Schiffmann Kartengrüße. Dazu Reisetagebuch, unpaginierter Anhang.

53 Die große Zeit der Bildpostkarten lag zwischen 1895 und 1914. Das Sammeln von Bildpostkarten entwickelte sich zu einer Leidenschaft, die alle Bevölkerungsgruppen umfasste, jedoch im bürgerlichen Mittelstand besonders stark vertreten war.

54 Zum Beispiel eine Ansichtskarte seines Vaters aus Hofgastein. Reisetagebuch: 65.

55 Zum Beispiel Reisetagebuch: 25 und 36. 
Seine Reiseaufzeichnungen machen auch deutlich, wie wichtig er sich genommen, und wie festhaltenswert er seine Karlsbader Erlebnisse eingeschätzt hat.

Krackowizer fügte sich in Karlsbad ein in sein gewohntes Netzwerk. Seine Aufzeichnungen zeigen, wie stark Wien orientiert der Kurort Karlsbad und viele der ihn Besuchenden waren. Pieter M. Judson hat in seiner Studie über das Imperium Habsburg beschrieben, „wie Institutionen des Reiches, administrative Praktiken und kulturelle Programme, an denen alle Staatsbürger teilhatten, dazu beitrugen, die lokalen Gesellschaften in jedem Winkel des Staatsgebietes zu formen" (Judson: 30). Durch diese „kollektiven Elemente" seien Erfahrungen vermittelt worden, „die sprachliche, konfessionelle und regionale Trennlinien wie auch zeitliche Grenzen überschritten“ (Judson: 30). „Dieses ,Wien“ der Gewohnheiten und Identifikationsangebote schuf vor allem im Bürgertum ein überregionales Netz von Identität, das die Zentralmacht möglicherweise stärker absichern konnte als Politik und Ökonomie dies vermochten." (Kos 1991b: 225)

September 2020

\section{Archivalische Quellen}

Oberösterreichisches Landesarchiv Linz:

Nachlass Krackowizer, Tagebücher, Reisetagebücher, Druckwerke, 1861-1957, Nr. 2.

Státní okresní archiv Karlovy Vary:

Gedenkbuch der Bezirkshauptmannschaft Karlsbad/Kronika okresního hejtmanství, 1888-1927, NAD 1, Inv. č. 16.

Stadtgemeinde Karlsbad: Karlsbader Kurliste 1909.

Stadtgemeinde Karlsbad: Karlsbader Kurliste 1910.

Sudetendeutsches Museum München:

Karlsbad 1909. Erinnerungsalbum, Ferdinand Krackowizers, Inv. Nr. 3809. 


\section{Literatur}

Adreß- und Geschäfts-Handbuch von Karlsbad und Umgebung 1906/07. 1906. Karlsbad: Verlag der Buchhandlung Rudolph Hengstenberg.

Antisemitische Bade-, Kur- u. Erholungsorte. 1909. Mitteilungen aus dem Verein zur Abwehr des Antisemitismus 19, 21/22, 26. Mai 1909: 163-164.

Augustin, Milan. 2010. Kurgästestatistik und Kurtaxe in Karlsbad In: Weigert, Ludwig J. (Hg.): Karlsbader Historische Schriften, Band 2. Karlovy Vary - München: Státní okresní archiv Karlovy Vary und Heimatverband der Karlsbader e.V.: 35-48.

Bajohr, Frank. 2003. „Unser Hotel ist judenfrei“. Bäder-Antisemitismus im 19. und 20. Jahrhundert. Frankfurt am Main: Fischer Taschenbuch Verlag. Bausinger, Hermann. 1991. Grenzenlos... Ein Blick auf den modernen Tourismus. In: Bausinger, Hermann - Beyrer, Klaus - Korff, Gottfried: Reisekultur. Von der Pilgerfahrt zum modernen Tourismus. München: C.H. Beck: 343-353.

Bericht der Handels- und Gewerbekammer in Eger 1859 an das k.k. Ministerium für Handel, Gewerbe und öffentliche Bauten über Handel, Industrie und Verkehrsmittel. 1859. Eger.

Bien, Helmut M. - Giersch, Ulrich. 1989. Reisen in die große weite Welt. Dortmund: Harenberg.

Burachovič, Stanislav. 2000. Karlozy Vary a jejich vlastivědné písemnictví. Prüvodce světem carlovarensiú. Karlovy Vary: Státní okresní archiv. Dipper, Rachel. 1999. „Einmal muss der Mensch ins Bad!“ Grüße aus Karlsbad und Marienbad. In: Gold, Helmut - Heuberger, Georg (Hg.): Abgestempelt. Fudenfeindliche Postkarten. Katalog zur Ausstellung „Abgestempelt. Judenfeindliche Postkarten“ im Jüdischen Museum Frankfurt am Main und im Museum für Post und Kommunikation Frankfurt am Main (14.4.1999-1.8.1999). (Kataloge der Museumsstiftung Post und Telekommunikation, Bd. 4). Heidelberg: Edition Braus: 194-204.

Dittmar, Peter. 1992. Die Darstellung der Fuden in der populären Kunst zur Zeit der Emanzipation. Hg. vom Zentrum für Antisemitismusforschung der Technischen Universität Berlin. München - London - New York - Paris: K. G. Saur.

[Ebner-Eschenbach, Marie von]. 1958. Aus Franzensbad. Sechs Episteln von keinem Propheten. Reprint der Ausgabe von 1858, hg. und komm. von Karlheinz Rossbacher. Wien: Österreichischer Bundesverlag. Exner, Wilhelm. 1929. Erlebnisse. Berlin - Heidelberg: Springer Verlag. Fendl, Elisabeth. 1995. Egerländer Volkskunde um 1900. Schweizerisches Archiv für Volkskunde 91, 2, 143-162. 
Fendl, Elisabeth. 2002. Mehr als ein Hotel: Das Pupp in Karlsbad. In: Knedlik, Manfred (Hg.): Spurensuche. Region. Grenzgänge. Festschrift für Franz Busl zum 70. Geburtstag. Pressath: Verlag der Buchhandlung Eckhard Bodner: 158-170.

Fendl, Elisabeth. 2011. Sie haben den englischen König bedient. Kellner in böhmischen Bädern. In: Köstlin, Konrad - Leonardi, Andrea Rösch, Paul (Hg.): Kellner und Kellnerinnen. Eine Kulturgeschichte. Beiträge zur internationalen Tagung „Darf es sonst noch was sein?" auf Schloss Trauttmansdorff vom 27. bis 29. Mai 2010. Milano: Silvana Editoriale: 55-77, Abb. 449-473.

Fleckles, Leopold. 1838. Karlsbad, seine Gesundbrunnen und Mineralbäder in geschichtlicher, topographischer, naturhistorischer und medicinischer Hinsicht. Stuttgart: J. Scheible's Buchhandlung.

Fuhs, Burkhard. 1992. Mondäne Orte einer vornehmen Gesellschaft. Kultur und Geschichte der Kurstädte 1700-1900. Hildesheim - Zürich - New York: Georg Olms Verlag.

Gintl, Franz. 1908. Guide to Carlsbad 1908. Vienna: Otto Maass. Gnirs, Anton. 1996. Topographie der historischen und kunstgeschichtlichen Denkmale in dem Bezirke Karlsbad (Prag 1933). München: Oldenbourg. Grundmann, Günter - Zechner, Christa. 2004. Sprudelstein lässt grüßen! Karlsbader Sprudelstein auf Ansichtskarten. Zdraví vás vř́delní kámen! Karlovarský vřídelní kámen na pohlednicích. In: Dittmar, Volker - Lehrberger, Gerhard: Der Sprudel macht den Stein - Schätze aus Karlsbad. Vrídlo dělá kámen - Poklady z Karlových Varů. Katalog zur gleichnamigen Ausstellung im Egerland-Museum Marktredwitz vom 23. Oktober 2004 bis 1. Mai 2005. Marktredwitz: Egerland-Museum Marktredwitz: 277-294.

Hanslick, Eduard. 1987. Aus meinem Leben. Kassel - Basel: Bärenreiter. Holm, Christiane. 2008. Montag Ich. Dienstag Ich. Mittwoch Ich. Versuch einer Phänomenologie des Diaristischen. In: @bsolut privat!? Vom Tagebuch zum Weblog. Kataloge der Museumsstiftung Post und Telekommunikation, Band 26. Heidelberg: Edition Braus im Wachter Verlag: 10-50.

Horst, Franz H. 1908. Österreichs Reiseländer. Kurorte, Heilstätten, Sommerfrischen und Winterstationen. Wien: Rudolf M. Rohrer.

Hubínek, Lubomír. 2013. Karlovarské lanovky. [2020-09-22]. Abrufbar unter: https://karlovarske-lanovky.webnode.cz.

Hürlimann, Annemarie. 1996. Schmutziger Leib. In: Mäßig und gefräßig. Wien: MAK- Österreichisches Museum für angewandte Kunst 1996: 110. John, Alois. 1907. Auf dem Grünberge. In: John, Alois.: Egerländer Heimatsbuch. Eger: Selbstverlag: 11-16. 
Judson, Pieter M. 2017. Habsburg. Die Geschichte eines Imperiums 17401918. München: Beck.

K. K. Statistische Zentralkommission. 1905. Gemeindelexikon von Böhmen, I. Teil, bearbeitet auf Grund der Ergebnisse der Volkszählung vom 31. Dezember 1900. Gemeindelexikon der im Reichsrate vertretenen Königreiche und Länder, IX. Böhmen. Wien: Verlag der K.K. Hofund Staatsdruckerei.

Klein, Peter K. 2006. Alltags-Antisemitismus im Kaiserreich. Das Beispiel der Judenspottkarten. In: Hoffmann, Andrea - Jeggle, Utz - Johler, Reinhard - Ulmer, Martin (Hg.): Die kulturelle Seite des Antisemitismus zwischen Aufklärung und Shoah. Studien und Materialien des LUI, Bd. 30; Tübinger kulturwissenschaftliche Gespräche, 3. Tübingen: Tübinger Vereinigung für Volkskunde: 125-171.

Köstlin, Konrad. 1988. Zur frühen Geschichte staatlicher Trachtenpflege in Bayern. In: Lehmann, Albrecht (Hg.): Sichtweisen der Volkskunde. Zur Geschichte und Forschungspraxis einer Disziplin. Berlin - Hamburg: Reimer: 301-319.

Köstlin, Konrad. 1992. „Durch diese Thuere schritt Goethe...“.

Denkmäler und Denkmalgänge in den Badeorten Nordwestböhmens. In: Haus der Heimat (Hg.): Das Egerländer Bäderdreieck von Weltruf. Stuttgart: $20-41$.

Köstlin, Konrad. 1994. Volkstümlicher Goethekult und die Nationalisierung des Egerlandes. Denkmalgänge als nationale Lehrpfade. In: Hahn, Gerhard - Weber, Ernst (Hg.): Zwischen den Wissenschaften. Beiträge zur deutschen Literaturgeschichte. Bernhard Gajek zum 65. Geburtstag. Regensburg: Verlag Friedrich Pustet: 36-47.

Kos, Wolfgang. 1991a. Distanz und Geselligkeit. Das Heilbad als soziale Experimentierbühne. In: Mattl-Wurm, Sylvia (Hg.): Das Bad. Körperkultur und Hygiene im 19. und 20. Fahrhundert. 142. Sonderausstellung des Historischen Museums der Stadt Wien, 23.3.1991-8.3.1992. Wien: $43-51$.

Kos, Wolfgang. 1991b. Zwischen Amüsement und Therapie. Der Kurort als soziales Ensemble. In: Lachmayer, Herbert - Mattl-Wurm, Sylvia - Gargerle, Christian (Hg.): Das Bad. Eine Geschichte der Badekultur im 19. und 20. Fahrhundert. Salzburg - Wien: Residenz Verlag: 220-236.

Krackowizer, Ferdinand - Berger, Franz (Hg.). 1931. Biographisches Lexikon des Landes Österreich ob der Enns. Passau - Linz: Institut für ostbaierische Heimatforschung.

Krajewski, Markus. 2008. Ask Jeeves. Der Diener als Informationszentrale. Recherche 3: 21-23. 
[Kretschmann, Karl Friedrich]. 1798. Reise nach den Bade-Oertern Karlsbad, Eger und Töplitz im Jahre 1797 in Briefen. Leipzig: Voß und Compagnie.

Kronser, Victor Nicolaus. 1861. Karlsbader Kur-Katechismus oder Neueste Beschreibung alles Wissenswerthen dieses Weltbades für Aerzte und Laien. Leipzig: Weber.

Leeb, Karl. 1902. Kurstatistik. In: Stadt Karlsbad (Hg.): Festschrift zur 74. Versammlung deutscher Naturforscher und Ärzte 1902. Karlsbad: Stadt Karlsbad: 589-630.

Lorenz, Reinhold. 1949. Bäderkultur und Kulturgeschichte. Forschungen über den Sozialcharakter der österreichischen Heilquellenorte. Archiv für österreichische Geschichte 117: 197-305.

Meyer, Silke. 2011. „Stimmt so“. Zur sozialen und kulturellen Semantik von Trinkgeld. In: Köstlin, Konrad - Leonardi, Andrea - Rösch, Paul (Hg.): Kellner und Kellnerin. Eine Kulturgeschichte. Beiträge zur internationalen Tagung „Darf es sonst noch was sein?" auf Schloss Trauttmansdorff vom 27. bis 29. Mai 2010. Milano: Silvana Editoriale: 259-275.

Oertl, Eduard - Ludwig, Karl - Becher, Karl. 1902. Die Bauentwicklung der Stadt Karlsbad. In: Stadt Karlsbad (Hg.): Festschrift zur 74.

Versammlung deutscher Naturforscher und Ärzte 1902. Karlsbad: Stadt Karlsbad: 181-217.

Oesterle, Günter. 2008. Die Intervalle des Tagebuchs - das Tagebuch als Intervall. In: @bsolut privat!? Vom Tagebuch zum Weblog. Kataloge der Museumsstiftung Post und Telekommunikation, Bd. 26. Heidelberg: Edition Braus: 100-103.

ÖBL. 1967. Österreichisches Biographisches Lexikon 1815-1950, Bd. 4. Stichwort Krackowizer: 183.

Polgar, Alfred. 1928. Städte, die ich nie erreichte. In: Ich bin Zeuge. Berlin: Ernst Rowohlt Verlag: 209-215.

Prein, Philipp. 2005. Bürgerliches Reisen im 19. Jahrhundert. Freizeit, Kommunikation und soziale Grenzen. (Kulturgeschichtliche Perspektiven, Band 3.) Münster: LIT Verlag.

Ries, Julius. 1843. Vieles über Carlsbad und Einiges über Oesterreich mit Bezugnahme auf dessen Industrie und Anschluß an den deutschen Zollverein. Leipzig: Joh. Friedr. Hartknoch.

Ruff, Josef. 1884. Illustrirtes Gesundheits-Lexicon. Ein populäres Handbuch für Jedermann. Straßburg: Schultz und Comp.

Ruff, Josef. 1887. Die Zuckerkrankheit (Diabetes mellitus): Ihre Erscheinung und ihre Behandlung. Tübingen: Verlag der Laupp'schen Buchhandlung.

Ruff, Josef. 1889. Die junge Mutter. Ärztliche Rathschläge und Winke für junge Frauen. Straßburg: Straßburger Druckerei und Verlagsanstalt. 
Ruff, Josef. 1894. Die Karlsbader Diät vor, während und nach der Kur: mit einleitenden Bemerkungen über Diät und Krankenpflege im Allgemeinen. Fellers Diätetische Führer, 3. Karlsbad - Leipzig: Verlag Hans Feller. Ruff, Josef (Bearb.). 1899. Karlsbad wie es war und wie es ist. Ein Führer für Kurgäste. Karlsbad: Stadtgemeinde Karlsbad.

Ruff, Josef (Bearb.). 1904. Karlsbad wie es war und wie es ist. Ein Führer für Kurgäste. Karlsbad: Stadtgemeinde Karlsbad.

Ruud, Peter. 1954. Heimat der Kellner. Internationale „Ober“ kamen aus dem Egerland. Wacht an der Miesa. Heimatbrieffür die Bezirke Mies, Pilsen, Staab, Tuschkau, Wiesengrund 48, 9: 358.

Schwarz, Hans-Peter (Hg.). 1988. Die Architektur der Synagoge. Stuttgart: Klett-Cotta.

Sebestyén, György. 1980. Die Kurpromenade oder die Erfindung der Kunstnatur. In: Große Welt reist ins Bad. Ausstellungskatalog Schloss Grafenegg, Passau: 36-42.

Schmitt, Michael. 1982. Palast-Hotels: Architektur und Anspruch eines Bautyps 1870-1920. Berlin: Gebrüder Mann Verlag.

Schönpflug, B. M. (Bearb.) 1875. Carlsbad und seine Umgebungen. Praktisches Handbuch für Reisende und Rathgeber für Curgäste. Berlin: Albert Goldschmidt.

Sommer, Hermann. 1999. Zur Kur nach Ems. Ein Beitrag zur Geschichte der Badereise von 1830 bis 1914. (Geschichtliche Landeskunde, Bd. 48.) Stuttgart: Franz Steiner Verlag.

Standseilbahnen in Karlsbad. In: Wikipedia. Die freie Enzyklopädie. [26.02.2020] https://de.wikipedia.org/wiki/Standseilbahnen_in_ Karlsbad.

Statistický lexikon obcí v Čechách. Úřední seznam míst podle zákona ze dne 14. dub. 1920. [Statistisches Lexikon der Gemeinden im Land. Offizielle Ortsliste]. Praha 1923.

Stürmer, Michael. 1990. Das ruhelose Reich. Deutschland 1866 bis 1918. Berlin: Siedler.

Telesko, Werner. 2014. Visualisierungsstrategien im Tourismus in der Spätphase der Habsburgermonarchie. Postkarten, Plakate und andere Bildmedien. In: Stachel, Peter - Thomsen, Martina (Hg.): Zwischen Exotik und Vertrautem. Zum Tourismus in der Habsburgermonarchie und ihren Nachfolgestaaten. Bielefeld: Transcript: 31-46.

Triendl-Zadoff, Mirjam. 2007a. Nächstes Fahr in Marienbad. Gegenwelten jüdischer Kulturen der Moderne. Jüdische Religion, Geschichte und Kultur, Band 6. Göttingen: Vandenhoeck \& Ruprecht.

Triendl-Zadoff, Mirjam. 2007b. Die Bügelfalte des Antisemitismus. Karlsbad, in der Sprache der Ambivalenz. In: Haas, Hanns - Hiebl, 
Ewald (Hg.): Politik vor Ort. Sinngebung in ländlichen und kleinstädtischen Lebenswelten. Jahrbuch für Geschichte des ländlichen Raumes 2007. Innsbruck - Wien - Bozen: StudienVerlag: 293-306.

Vybíral, Jindřich. 2007. Junge Meister. Architekten aus der Schule Otto

Wagners in Mähren und Schlesien. (ARS VIVA, Band 11.) Wien - Köln Weimar: Böhlau.

Wenzel, Maria. 1991. Palasthotels in Deutschland. Untersuchungen zu einer Bauaufgabe im 19. und frühen 20. Fahrhundert. Hildesheim - Zürich New York: Olms.

Winter, Max. 1901. Die Kehrseite der Medaille. Arbeiter-Zeitung Nr. 204, 28.7.1901.

Zerlik, Otto. 1983. Vom japanischen Pavillon in Karlsbad-Fischern. Fahrbuch der Egerländer 32: 83-86.

Zibermayr, Ignaz. 1935. Ferdinand Krackowizer . Jahrbuch des oberösterreichischen Musealvereines 86: 92-95.

Zídková, Anna. 1996. Fellner a Helmer v Karlozých Varech. Karlovy Vary: Sazba Median a. s. 